\title{
INTEGRATION AND NONLINEAR TRANSFORMATIONS IN HILBERT SPACE
}

\author{
BY \\ LEONARD GROSS( $\left({ }^{1}\right)$
}

The purpose of this paper is to generalize to Hilbert space a well known theorem of Jacobi on the transformation of integrals under a change of coordinates in $E_{n}$. In the process we develop some of the relevant integration theory over Hilbert space. Among the various theories $[14 ; 3 ; 4 ; 5 ; 9 ; 10]$, of integration over a real Hilbert space $H$ we shall utilize the one $[14 ; 3 ; 4 ; 5]$, which seems most relevant to the quantum theory of fields and which is intimately connected with the Wiener integral. The formulation of the theory which we adopt is given in Segal [14, pp. 116-118] and we refer the reader to this paper for details. In outline this formulation consists in associating with $H$ in an invariant way a probability space $(S, m)$ and a map $f \rightarrow \tilde{f}$ which assigns a measurable function $\tilde{f}$ on $(S, m)$ to each tame function $f$ on $H$, a tame function being one which, roughly speaking, depends only on a finite number of coordinates in $H$. The theory of integration over $H$ is then the theory of integration over $(S, m)$ with particular emphasis on those questions which arise from the relation of $H$ to $(S, m)$. In case $H$ is real $L^{2}(0,1)$ the probability space may be taken to be Wiener space.

We first describe (Theorem 1) a class of continuous functions on $H$ which are determined by their continuity properties alone and which correspond in a natural way to measurable functions on $(S, m)$, i.e., functions $f$ other than tame functions for which $\bar{f}$ makes sense. The type of continuity involved plays a central role in the remainder of the paper and generalizes ordinary continuity in $E_{n}$ in the relevant way for the purposes of integration theory. Theorem 2 relates the convergence of sequences of these functions to convergence in probability of the associated measurable functions. Theorem 3 is a density theorem for the group of automorphisms of the algebra $L^{\infty}(S, m)$ and provides a background for the approximation method used in the following theorem. Theorem 4 is a generalization of Jacobi's theorem for a transformation of the form $T=I+K$ where $I$ is the identity operator and $K$ is nonlinear, small and smooth. In Theorem 5 we avoid the smallness requirement by considering a one-parameter group of nonlinear transformations on $H$ on whose generator sufficient conditions are imposed which ensure that the elements of the group satisfy a Jacobi-like theorem. The case of linear transformations has already been investigated by Segal [16].

Presented to the Society, October 25, 1958; received by the editors March 2, 1959.

(1) This is largely the author's doctoral dissertation at The University of Chicago, 1957. The author wishes to express his gratitude to Professor I. E. Segal under whose direction the thesis was written. 
Our results may be compared with those of Cameron and Martin [2]. We compare them briefly in the last example of the paper.

1. Measurable functions on Hilbert space. We follow Segal [14] in making

Definition 1. A weak distribution on a real topological linear space, $L$, is an equivalence class of linear maps from the dual space, $L^{*}$, of continuous linear functionals on $L$ to real valued random variables on a probability space where two such maps, $y \rightarrow n(y)$ and $y \rightarrow m(y)$, are equivalent if for any finite set of vectors, $y_{1}, \cdots, y_{k}$, in $L^{*}$ the two sets of random variables, $n\left(y_{1}\right), \cdots, n\left(y_{k}\right)$ and $m\left(y_{1}\right), \cdots, m\left(y_{k}\right)$, have the same joint distributions in $k$-space.

The relevant integration theory with respect to a weak distribution on a linear space is described in [14, Part II, §2, pp. 116-118]. We shall be concerned with a real Hilbert space, $H$, and we shall identify $H$ and $H^{*}$ in the usual way. The distribution will be for the most part the canonical normal distribution with parameter $c$, i.e., that distribution, $n$, which is uniquely determined by (1) for any vector $x$ in $H n(x)$ is normally distributed with mean zero and variance $c\|x\|^{2}$, (2) $n$ takes orthogonal vectors into independent random variables. A tame function on $H$ is a function of the form $f(x)$ $=\phi(P x)$ where $P$ is a finite dimensional projection on $H$ and $\phi$ is a complex valued Baire function on the range of $P$. By a measurable function on $H$ we shall mean any random variable on the probability space mentioned in Definition 1 which is measurable with respect to the smallest Borel field with respect to which all of the random variables $n(x)$ are measurable. Furthermore we shall take for this probability space the particular one arrived at by the process described in [14, p. 117] unless otherwise stated. A tame function on $H$ determines a "measurable tame function" on the probability space as in [14] and we shall frequently identify the tame function with the corresponding measurable function. We shall also consider the $L^{p}$ space over the probability space and refer to it as $L^{p}(H, c)$ or simply $L^{p}(H)$ since the parameter $c$ will be fixed throughout this paper. In this section we describe a class of continuous functions on $H$ which correspond in a natural way to measurable functions on $H$ and we relate the convergence properties of these functions on $H$ to convergence in probability of the corresponding measurable functions.

Denoting by $H_{0}$ the Hilbert space, $H$, in its norm topology we define a topology, $H_{2}$, as the weakest topology on $H$ such that all Hilbert-Schmidt operators on $\mathrm{H}$ are continuous from $\mathrm{H}_{2}$ to $\mathrm{H}_{0} . \mathrm{H}_{2}$ is a locally convex topological vector space with basic open sets of the form $U\left(x_{0}, A\right)=\left\{x:\left\|A\left(x-x_{0}\right)\right\|<1\right\}$ where $A$ is a Hilbert-Schmidt operator. It is readily verified that these form a basis using the fact that if $A$ and $B$ are Hilbert-Schmidt operators so is $\left(A^{*} A+B^{*} B\right)^{1 / 2}$. We denote the Hilbert-Schmidt norm, (trace $\left.A^{*} A\right)^{1 / 2}$, by $\|A\|_{2}$. 
Definitron 2. A function, $f$, from $H$ to a topological vector space is uniformly continuous near zero in the topology $\mathrm{H}_{2}$ if there exists a sequence, $A_{n}$, of Hilbert-Schmidt operators such that $\left\|A_{n}\right\|_{2} \rightarrow 0$ and such that $f$ is uniformly continuous in the topology $H_{2}$ on each of the sets $U_{n}$ $=\left\{x:\left\|A_{n} x\right\|<1\right\}$.

REMARK. If $H$ is finite dimensional then the preceding definition is equivalent to ordinary continuity. Indeed if $f$ is continuous then it is uniformly continuous on the sphere of radius $n$ about the origin, i.e., one may take $A_{n}=n^{-1} I$ where $I$ is the identity operator on $H$.

Theorem 1. Let $f$ be a complex valued function on $H$ which is uniformly continuous near zero in the topology $\mathrm{H}_{2}$. Denote by $\mathcal{F}$ the directed set of finite dimensional projections on $H$ directed by inclusion of the ranges and let I denote the identity operator on $H$. The net $\{f \circ P, P$ in $F\}$ of measurable tame functions converges in probability as $P \rightarrow I$ through $\mathcal{F}$ to a measurable function $\tilde{f}$.

Lemma 1.1. Let $A$ be a finite dimensional operator on $H$. Then $\|A x\|^{2}$ is an integrable tame function with integral $E\left(\|A x\|^{2}\right)=c$ trace $A^{*} A$.

Proof. Let $G$ be the orthogonal complement of the null space of $A^{*} A$. Then $G$ is finite dimensional and $\|A x\|^{2}$ is a tame function based on $G$. Let $x_{1}, \cdots, x_{k}$ be an orthonormal basis of $G$ such that $A^{*} A x_{i}=s_{i} x_{i}, i=1, \cdots, k$, where the $s_{i}$ are real numbers. Putting $x=\sum t_{i} x_{i}$ we have $E\left(\|A x\|^{2}\right)$ $=E\left(\left(A^{*} A x, x\right)\right)=(2 \pi c)^{-k / 2} \int \sum s_{i} t_{i}^{2} \exp \left(-\sum_{i=1}^{k} t_{i}^{2} / 2 c\right) d t_{1} \cdots d t_{k}=c \sum_{i=1}^{k} s_{i}=c$ trace $\left(A^{*} A\right)$. Q.E.D.

Lemma 1.2. Let $A$ be a Hilbert-Schmidt operator. Then the net $\{\|A P x\|:$ $P$ in $\mathcal{F}\}$ of measurable tame functions converges in $L^{2}(H, c)$ as $P \rightarrow I$ through $\mathcal{F}$ to a measurable function $\|A(\cdot)\|$ with expectation $E\left(\|A(\cdot)\|^{2}\right)=c\|A\|_{2}^{2}$.

Proof. If $P$ and $Q$ are in $\mathcal{F}$ then $\|A P x\|-\|A Q x\|\|\leqq\| A(P-Q) x \|$. Hence $E\left((\|A P x\|-\|A Q x\|)^{2}\right) \leqq E\left(\|A(P-Q) x\|^{2}\right)=c\|A(P-Q)\|_{2}^{2}$. Let $x_{1}, x_{2}, \cdots$ be an orthonormal basis of the carrier of $A^{*} A$ such that $A^{*} A x_{i}=s_{i} x_{i}, \quad i$ $=1,2, \cdots$ and denote by $P_{n}$ the projection onto $\operatorname{span}\left(x_{1}, \cdots, x_{n}\right)$. If $P \geqq P_{n}$ and $Q \geqq P_{n}$ then $\|A(P-Q)\|_{2} \leqq\|A(I-P)\|_{2}+\|A(I-Q)\|_{2}$ $=\left\|A\left(I-P_{n}\right)(I-P)\right\|_{2}+\left\|A\left(I-P_{n}\right)(I-Q)\right\|_{2} \leqq 2\left\|A\left(I-P_{n}\right)\right\|_{2}=2 \sum_{i>n} s_{i}$ which approaches zero as $n \rightarrow \infty$. Hence $\|A(P-Q)\|_{2} \rightarrow 0$ as $P, Q \rightarrow I$ through $\mathcal{F}$ and it follows that $\|A P x\|$ converges in $L^{2}(H, c)$. Further, $E\left(\|A(\cdot)\|^{2}\right)$ $=\lim _{P \rightarrow I} E\left(\|A P x\|^{2}\right)=c \lim _{P \rightarrow I}\|A P\|_{2}^{2}=c\|A\|_{2}^{2}$. Q.E.D.

Proof of theorem. Given $\epsilon>0$ and $\delta>0$ we shall construct a projection, $P_{0}$, in $\mathcal{F}$ such that if $P \geqq P_{0}$ and $Q \geqq P_{0}$ and $P$ and $Q$ are in $\mathcal{F}$ then $\operatorname{Prob}(|f \circ P-f \circ Q| \geqq \epsilon)<\delta$. Let $A_{n}$ be a sequence of Hilbert-Schmidt operators such that $\left\|A_{n}\right\|_{2} \rightarrow 0$ and such that $f$ is uniformly continuous in the topology $H_{2}$ on each of the sets $\left\{x:\left\|A_{n} x\right\|<1\right\}$. By Lemma 1.2 the measurable functions $\left\|A_{n}(\cdot)\right\|$ converge to zero in $L^{2}(H)$ hence also in probability. 
Let $n$ be an integer which is fixed hereafter such that $\operatorname{Prob}\left(\left\|A_{n}(\cdot)\right\| \geqq 1 / 2\right)$ $<\delta / 6$. By Lemma 1.2 the net of tame functions $\left\{\left\|A_{n} P x\right\|: P\right.$ in $\left.\mathcal{F}\right\}$ converges in probability to $\left\|A_{n}(\cdot)\right\|$. There exists a projection, $R$, in $\mathcal{F}$ such that if $P$ is in $\mathcal{F}$ and $P \geqq R$ then $\operatorname{Prob}\left(\left|\left\|A_{n}(\cdot)\right\|-\left\|A_{n} P x\right\|\right| \geqq 1 / 2\right)<\delta / 6$. Hence $\operatorname{Prob}\left(\left\|A_{n} P x\right\| \geqq 1\right)<\delta / 3$ whenever $P$ is in $\mathcal{F}$ and $P \geqq R$.

By the uniform continuity of $f$ on $\left\{x:\left\|A_{n} x\right\|<1\right\}$ there exists a HilbertSchmidt operator, $A$, such that $|f(x)-f(y)|<\epsilon$ whenever $\left\|A_{n} x\right\|<1,\left\|A_{n} y\right\|$ $<1$ and $\|A(x-y)\|<1$. In the proof of Lemma 1.2 it is shown that there exists a projection, $S$, in $\mathcal{F}$ such that if $P$ and $Q$ are in $\mathcal{F}$ and $P \geqq S$ and $Q \geqq S$ then $\|A(P-Q)\|_{2}<(\delta / 3 c)^{1 / 2}$ so that $\operatorname{Prob}(\|A(P-Q) x\| \geqq 1)<\delta / 3$. Let $P_{0}$ $=R \bigvee S$, the supremum being taken in the lattice $\mathcal{F}$. As $|f(P x)-f(Q x)|<\epsilon$ whenever $\left\|A_{n} P x\right\|<1,\left\|A_{n} Q x\right\|<1$ and $\|A(P-Q) x\|<1$ it follows that if $P$ and $Q$ are in $\mathfrak{F}$ and $P \geqq P_{0}$ and $Q \geqq P_{0}$ then $\operatorname{Prob}(|f(P x)-f(Q x)| \geqq \epsilon)$ $\leqq \operatorname{Prob}\left(\left\|A_{n} P x\right\| \geqq 1\right)+\operatorname{Prob}\left(\left\|A_{n} Q x\right\| \geqq 1\right)+\operatorname{Prob}(\|A(P-Q) x\| \geqq 1)<3 \delta / 3=\delta$ proving the assertion in the first line of the proof.

Letting $\epsilon=\delta=1 / k, k=1,2, \cdots$ we obtain projections $P_{k}$ in $\mathcal{F}$ as above such that if $P$ and $Q$ are in $\mathcal{F}$ and $P \geqq P_{k}$ and $Q \geqq P_{k}$ then

$$
\operatorname{Prob}(|f(P x)-f(Q x)| \geqq 1 / k)<1 / k .
$$

The $P_{k}$ may be assumed increasing. Denoting by $\bar{f}$ the limit in probability of the sequence $f \circ P_{k}$ it is readily verified that $\operatorname{Prob}(|f(P x)-\tilde{f}| \geqq \epsilon) \rightarrow 0$ as $P \rightarrow I$ through $\mathcal{F}$.

COROLlaRY 1.1. Let $f$ be a complex valued function on $H$ which is uniformly continuous near zero in the topology $H_{2}$. Then the corresponding measurable function $\tilde{f}$ is a null function (i.e. equals zero almost everywhere) if and only if $f$ is identically zero on $H$.

Lemma 1.1.1. Let $C$ be a Hilbert-Schmidt operator on $H$ and let $x_{0}$ be in $H$. Denote by $\tilde{g}$ the measurable function corresponding to the function $g(x)$ $=\left\|C\left(x-x_{0}\right)\right\|$. If $a>0$ then $\operatorname{Prob}(\tilde{g}<a)>0$.

Proof. It should be remarked that $g$ is uniformly continuous in $\mathrm{H}_{2}$ so that $\tilde{g}$ exists. By Theorem 3 of [16] the canonical normal distribution is quasiinvariant under translations hence it suffices to prove the lemma for the case $x_{0}=0$. We may assume that $C^{*}=C \geqq 0$ by replacing $C$ by $\left(C^{*} C\right)^{1 / 2}$. By the proof of Lemma 1.2 we may write $\tilde{g}=\lim$ in $\operatorname{prob}_{n \rightarrow \infty}\left\|C P_{n} x\right\|$ where $P_{n}$ is an increasing sequence of finite dimensional spectral projections of $C$ such that the span of the ranges of the $P_{n}$ is the carrier of $C$. Put $h_{m}(x)=\left\|C\left(I-P_{m}\right) x\right\|$. Then $\tilde{g}^{2}=\lim$ in $\operatorname{prob}_{n \rightarrow \infty}\left(\left\|C P_{m} x\right\|^{2}+h_{m}^{2}\left(P_{n} x\right)\right)=\left\|C P_{m}(\cdot)\right\|^{2}+\tilde{h}_{m}^{2}$. Since $E\left(\tilde{h}_{m}^{2}\right)$ $=c\left\|C\left(I-P_{m}\right)\right\|_{2}^{2}$ we may choose $m$ so large that $E\left(\tilde{h}_{m}^{2}\right)<a^{2} / 4$. Then $\operatorname{Prob}\left(\tilde{h}_{m}^{2}<a^{2} / 2\right) \geqq 1 / 2$. Further $\operatorname{Prob}\left(\left\|C P_{m}(\cdot)\right\|^{2}<a^{2} / 2\right)$ is equal to the normalized Gaussian measure of an open ellipsoid in a finite dimensional space. Hence $\operatorname{Prob}\left(\left\|C P_{m}(\cdot)\right\|^{2}<a^{2} / 2\right)>0$. As $\left\|C P_{m}(\cdot)\right\|^{2}$ and $\tilde{h}_{m}^{2}$ are independent random variables it follows that 


$$
\begin{aligned}
\operatorname{Prob}\left(\tilde{g}^{2}<a^{2}\right) & \geqq \operatorname{Prob}\left(\tilde{h}_{m}^{2}<a^{2} / 2,\left\|C P_{m}(\cdot)\right\|^{2}<a^{2} / 2\right) \\
& =\operatorname{Prob}\left(\tilde{h}_{m}^{2}<a^{2} / 2\right) \cdot \operatorname{Prob}\left(\left\|C P_{m}(\cdot)\right\|^{2}<a^{2} / 2\right)>0 .
\end{aligned}
$$

Proof of corollary. We shall show that if there is a vector, $x_{0}$, in $H$ such that $f\left(x_{0}\right) \neq 0$ then $\tilde{f}$ is not a null function. We may assume that $f\left(x_{0}\right)=a>0$. There exists a Hilbert-Schmidt operator, $A$, such that $\left\|A x_{0}\right\|=b<1$ and such that $f$ is uniformly continuous in the topology $H_{2}$ on the set $V=\{x\|A x\|<1\}$. There exists a Hilbert-Schmidt operator, $B$, such that $|f(x)-f(y)|<a / 3$ whenever $x$ and $y$ are in $V$ and $\|B(x-y)\|<1$. The operator

$$
C=(1-b)^{-1}\left(A^{*} A+B^{*} B\right)^{1 / 2}
$$

is Hilbert-Schmidt and if $\left\|C\left(x-x_{0}\right)\right\|<1$ then $\|A x\| \leqq\left\|A x_{0}\right\|+\left\|A\left(x-x_{0}\right)\right\|$ $<b+1-b=1$ and also $\left\|B\left(x-x_{0}\right)\right\|<1-b \leqq 1$. Hence if $\left\|C\left(x-x_{0}\right)\right\|<1$ then $f(x)=f\left(x_{0}\right)+\left(f(x)-f\left(x_{0}\right)\right) \geqq a-a / 3=2 a / 3$. Putting $g(x)=\left\|C\left(x-x_{0}\right)\right\|$ we observe that $g$ is uniformly continuous in the topology $H_{2}$ and denote by $\tilde{g}$ the corresponding measurable function. Let $d=\operatorname{Prob}(\tilde{g}<1 / 2)$. By Lemma 1.1.1 $d>0$. There exists a finite dimensional projection, $P$, having the properties that $x_{0}$ is in its range, that $\operatorname{Prob}(|\tilde{g}-g \circ P| \geqq 1 / 2)<d / 3$ and that $\operatorname{Prob}(|\tilde{f}-f \circ P| \geqq a / 3)<d / 3$. Note that if $g(P x)<1$ then $f(P x) \geqq 2 a / 3$. Thus $\operatorname{Prob}(\tilde{f} \geqq a / 3) \geqq \operatorname{Prob}(f \circ P \geqq 2 a / 3)-\operatorname{Prob}(|\bar{f}-f \circ P| \geqq a / 3) \geqq \operatorname{Prob}(g \circ P<1)$ $-d / 3 \geqq \operatorname{Prob}(\tilde{g}<1 / 2)-\operatorname{Prob}(|\tilde{g}-g \circ P| \geqq 1 / 2)-d / 3 \geqq d / 3$.

THEOREM 2. Let $\left\{f_{n}\right\}$ be a sequence of complex valued functions on a real Hilbert space, $H$, which are uniformly continuous near zero in the topology $\mathrm{H}_{2}$. Let $B_{k}$ be a sequence of Hilbert-Schmidt operators on $H$ such that $\left\|B_{k}\right\|_{2} \rightarrow 0$ as $k \rightarrow \infty$ and assume that $f_{n}$ converges to a function, $f$, uniformly on the sets $\left\{x:\left\|B_{k} x\right\|<1\right\}$. Then $f$ is uniformly continuous near zero in the topology $\mathrm{H}_{2}$ and denoting by $\tilde{f}_{n}, \tilde{f}$ the corresponding measurable functions we have $\tilde{f}=$ limit in probability $\tilde{f}_{n}$.

Proof. There exist Hilbert-Schmidt operators $A_{n, k}$ such that, for each $n, f_{n}$ is uniformly continuous in $H_{2}$ on the sets $\left\{x:\left\|A_{n, k} x\right\|<1\right\}, k=1,2, \cdots$ and $\left\|A_{n, k}\right\|_{2} \rightarrow 0$ as $k \rightarrow \infty$. For each pair of positive integers, $j, n$ there exists an integer $k(j, n)$ such that $\left\|A_{n, k(j, n)}\right\|_{2}^{2} \leqq j^{-2} 2^{-n}$. Let

$$
C_{j}=\left(\sum_{n \geq 1} A_{n, k(j, n)}^{*} A_{n, k(j, n)}\right)^{1 / 2} .
$$

The sum converges in Hilbert-Schmidt norm and in fact $\left\|C_{j}\right\|_{2}<j^{-1}$. Further, $\left\|C_{j} x\right\|<1$ implies $\left\|A_{n, k(j, n)} x\right\|<1$ for all $n$, hence $f_{n}$ is uniformly continuous in $H_{2}$ on $\left\{x:\left\|C_{j} x\right\|<1\right\}$ for each $n$ and $j$. Finally put $D_{j}=\left(C_{j}^{2}+B_{j}{ }^{*} B_{j}\right)^{1 / 2}$. Then $\left\|D_{j}\right\|_{2} \rightarrow 0, f_{n}$ is uniformly continuous in $H_{2}$ on the sets $V_{j}=\left\{x:\left\|D_{j} x\right\|<1\right\}$ and $f_{n} \rightarrow f$ uniformly on $V_{j}$. It follows that $f$ is uniformly continuous in $H_{2}$ on each $V_{j}$ proving the first assertion of the theorem. 
Given $\epsilon>0$ and $\delta>0$ we shall show that there exists an integer $N$ such that $\operatorname{Prob}\left(\left|\tilde{f}_{n}-\tilde{f}\right| \geqq \epsilon\right)<\delta$ whenever $n \geqq N$. Fix $j$ so large that $\left\|D_{j}\right\|_{2}^{2}<(\delta / 24 c)$. Using the notation and result of Lemma 1.2 it follows from $E\left(\left\|D_{j}(\cdot)\right\|^{2}\right)$ $<\delta / 24$, that $\operatorname{Prob}\left(\left\|D_{j}(\cdot)\right\| \geqq 1 / 2\right)<\delta / 6$ and that there exists a projection $R$ in $\mathcal{F}$ such that if $P$ is in $\mathcal{F}$ and $P \geqq R$ then $\operatorname{Prob}\left(\left|\left\|D_{j}(\cdot)\right\|-\left\|D_{j} P x\right\|\right| \geqq 1 / 2\right)$ $<\delta / 6$. Hence if $P$ is in $\mathcal{F}$ and $P \geqq R$ then $\operatorname{Prob}\left(\left\|D_{j} P x\right\| \geqq 1\right)<\delta / 3$. Choose $N$ so that $\left|f_{n}(x)-f(x)\right|<\epsilon / 3$ whenever $x$ is in $V_{j}$ and $n \geqq N$. Fix $n \geqq N$. There exists a projection, $S$, in $\mathcal{F}$ such that if $P$ is in $\mathcal{F}$ and $P \geqq S$ then

$$
\operatorname{Prob}(|\tilde{f}-f(P x)| \geqq \epsilon / 3)<\delta / 3
$$

and

$$
\operatorname{Prob}\left(\left|f_{n}(P x)-\tilde{f}_{n}\right| \geqq \epsilon / 3\right)<\delta / 3 \text {. }
$$

Now

$$
\begin{aligned}
\operatorname{Prob}\left(\left|\tilde{f}_{n}-\tilde{f}\right|\right. & \geqq \epsilon) \leqq \operatorname{Prob}\left(\left|\tilde{f}_{n}-f_{n}(P x)\right| \geqq \epsilon / 3\right) \\
& +\operatorname{Prob}\left(\left|f_{n}(P x)-f(P x)\right| \geqq \epsilon / 3\right)+\operatorname{Prob}(|f(P x)-\tilde{f}| \geqq \epsilon / 3) .
\end{aligned}
$$

Choose $P \geqq R \bigvee S$. Then the first and third terms on the right of the last inequality are each less than $\delta / 3$ while $\left|f_{n}(P x)-f(P x)\right| \geqq \epsilon / 3$ implies $\left\|D_{j} P x\right\| \geqq 1$ so that the middle term is also less than $\delta / 3$. Hence $\operatorname{Prob}\left(\left|\tilde{f}_{n}-\tilde{f}\right| \geqq \epsilon\right)<\delta$. Q.E.D.

Examples. (1) Let $g$ be a complex valued function on $H$ which is uniformly continuous in the norm topology on bounded sets. If $A$ is a HilbertSchmidt operator then the function $f(x)=g(A x)$ is uniformly continuous near zero in the topology $H_{2}$. One may take $A_{n}=n^{-1} A$ to satisfy Definition 2 .

(2) A uniformly continuous function in $\mathrm{H}_{2}$ is uniformly continuous near zero in $H_{2}$. Take all $A_{n}=0$.

(3) A tame function, $f$, on $H$ which is continuous in the norm topology is uniformly continuous near zero in $H_{2}$. If $f$ is based on the range of the finite dimensional projection, $P$, then we may take $A_{n}=n^{-1} P$. A uniform limit of such continuous tame functions is uniformly continuous near zero in $\mathrm{H}_{2}$ by Theorem 2 . The corresponding measurable function is then not only a limit in probability of measurable tame functions but also a uniform limit of such functions.

REMARK. Theorem 1 shows that a bounded complex valued function, $f$, which is uniformly continuous near zero in the topology $\mathrm{H}_{2}$ is an invariant function in the sense of Friedrichs and Shapiro [4]. The integral of $f$ may be defined by $\int_{H} f=E(\tilde{f})$.

2. Automorphisms of the bounded algebra of $H$. Let $(S, \mu)$ be a probability space. By the bounded algebra of $(S, \mu)$ we shall mean the algebra, $\mathfrak{T}$, of bounded complex valued measurable functions on $S$ modulo the ideal of null functions. If $f$ is in $\mathfrak{T}$ we denote by $M_{f}$ the bounded linear operator on $L^{2}(S, \mu)$ which consists of multiplication by $f$. To each automorphism, $\phi$, of 
Th we shall associate a unique unitary transformation, $U$, of $L^{2}(S, \mu)$ such that $U M_{f} U^{-1}=M_{\phi(f)}$ for all $f$ in $\mathscr{T}$ and having the properties

$$
U(1)>0 \text { a.e., } U(f) U(g)=U(1) U(f g) \text { for all } f \text { and } g \text { in } \mathfrak{T} \text {. }
$$

Conversely every unitary transformation on $L^{2}(S, \mu)$ satisfying (A) corresponds to a unique automorphism of $\mathfrak{T}$. To establish this correspondence suppose first that $\phi$ is an automorphism of $\mathfrak{T}$. By Theorem 5 of [13] $\phi$ is a homeomorphism of $\mathfrak{T}$ in the weak topology which $\mathfrak{T}$ inherits as the dual space to $L^{1}(S, \mu)$. It follows that if $C_{R}$ denotes the characteristic function of the measurable set, $R$, then the set function $\nu(R)=\int_{S} \phi^{-1}\left(C_{R}\right) d \mu$ is a countably additive probability measure which is absolutely continuous with respect to $\mu$. Denoting by $h$ the Radon-Nikodym derivative $d \nu / d \mu$ we observe that $h>0$ a.e. with respect to $\mu$ and $\int_{s} h d \mu=1$. Thus

$$
\int_{S} \phi^{-1}(f) d \mu=\int_{S} f h d \mu
$$

when $f$ is a characteristic function. (1) also holds when $f$ is a simple function and as an automorphism of $\mathfrak{T}$ is continuous in the $L^{\infty}$ topology of $\mathfrak{T}$ and as $h$ is integrable it follows that (1) is valid for all $f$ in $\mathfrak{T}$. Now put $U(f)=\phi(f) h^{1 / 2}$. It is not difficult to verify, using (1), that $U$ is unitary on bounded functions and extends uniquely to a unitary transformation on $L^{2}(S, \mu)$ which we shall also denote by $U$. Note that $U(1)=\phi(1) h^{1 / 2}>0$ a.e. and if $f$ and $g$ are in $T$ then $U(f) U(g)=\phi(f) \phi(g) h=\phi(f g) h=U(1) U(f g)$. Further, if $U^{-1} g$ is in $\mathfrak{T}$ then $U M_{f} U^{-1} g=(U(f) / U(1)) U U^{-1} g=\phi(f) g=M_{\phi(f)} g$.

Conversely if (A) holds for a unitary transformation, $U$, on $L^{2}(S, \mu)$ then put $\phi(f)=U(f) / U(1)$ for $f$ in $\mathfrak{T}$. If $f$ and $U^{-1} g$ are in $\mathbb{T}$ then $U M_{f} U^{-1} g$ $=U\left(f U^{-1} g\right)=(U(f) / U(1)) U U^{-1} g=\phi(f) g$. It follows from the density of $U(\mathfrak{T T})$ in $L^{2}(S, \mu)$ that $U M_{f} U^{-1}=M_{\phi(f)}$ and that $\|\phi(f)\|_{\infty}=\|f\|_{\infty}$. Hence $\phi$ is defined on $\mathfrak{T}$ and (A) implies further that $\phi$ is a homomorphism. To see that $\phi$ is onto observe that if $f$ and $g$ are in $\mathscr{T}$ then $\left(U^{-1} M_{g} U\right) M_{f}$ $=U^{-1} M_{\theta} M_{\phi(f)} U=U^{-1} M_{\phi(f)} M_{\theta} U=M_{f}\left(U^{-1} M_{\theta} U\right)$. Hence $U^{-1} M_{\theta} U$ commutes with the maximal abelian self-adjoint algebra $\left\{M_{f}: f\right.$ in $\left.\mathfrak{M}\right\}$, implying that $U^{-1} M_{\theta} U=M_{k}$ for some $k$ in $\mathfrak{T}$. Thus $M_{\theta}=U M_{k} U^{-1}=M_{\phi(k)}$ and $g=\phi(k)$. $U$ will be called the unitary associated with the automorphism $\phi$. Note that the map $\phi \rightarrow U$ is an algebraic isomorphism of the automorphism group of $\mathfrak{T}$ onto the group of unitaries satisfying (A).

We continue to deal in this section with the canonical normal distribution with parameter $c$ on a real Hilbert space, $H$. By the bounded algebra of $H$ we shall mean the bounded algebra of the associated probability space. In case $H$ is finite dimensional it is known that every automorphism of its bounded algebra arises from a measurable transformation, $T$, of $H$ by means of the relation $\phi(f)=f \circ T$ where $T$ is one to one and onto except for sets of measure zero, whose inverse is also measurable and for which the measure 
$m \circ T^{-1}$ is equivalent to $m$ in the sense of absolute continuity, $m$ being Lebesgue measure on $H$. Conversely every such transformation, $T$, gives rise to an automorphism of the bounded algebra in this way. It is the purpose of this section to show that in case $H$ is infinite dimensional every automorphism of the bounded algebra of $H$ may be approximated in the sense of the strong operator topology on the associated unitaries by automorphisms arising from transformations of $H$ which act essentially in a finite dimensional subspace of $H$.

If $K$ is a closed subspace of $H$ and $f$ and $g$ are bounded tame functions on $K$ and $K^{\perp}$ respectively then $f g$ is a bounded tame function on $H$ and the map $f \otimes g \rightarrow f g$ extends to a unique unitary transformation from $L^{2}(K) \otimes L^{2}\left(K^{\perp}\right)$ onto $L^{2}(H)$. We shall identify $L^{2}(H)$ with $L^{2}(K) \otimes L^{2}\left(K^{\perp}\right)$ when convenient by means of this unitary transformation.

Definition 3. A measurable function, $f$, in $L^{2}(H)$ is based on a closed subspace, $K$, of $H$ if there is a function, $g$, in $L^{2}(K)$ such that $f=g \otimes 1$ in the decomposition $L^{2}(H)=L^{2}(K) \otimes L^{2}\left(K^{\perp}\right)$. A bounded linear operator $A$ on $L^{2}(H)$ will be said to be based on a closed subspace $K$ of $H$ if there is a bounded linear operator, $B$, on $L^{2}(K)$ such that $A=B \otimes I$ in the decomposition $L^{2}(H)$ $=L^{2}(K) \otimes L^{2}\left(K^{\perp}\right)$.

TheOReм 3. Let $H$ be a real separable Hilbert space and let $U$ be a unitary transformatiou on $L^{2}(H)$ associated with an automorphism of the bounded algebra of $H$. Let $x_{1}, x_{2}, \cdots$ be an orthonormal basis of $H$ and put $H_{n}$ $=\operatorname{span}\left(x_{1}, \cdots, x_{n}\right)$. Then there exists a sequence of unitary transformations, $U_{n}$, on $L^{2}(H)$, associated with automorphisms, such that $U_{n}$ is based on $H_{n}$ and such that $U_{n}$ converges to $U$ strongly.

Proof. Let $R_{n}$ be the real line with element of measure

$$
(2 \pi c)^{-1 / 2} \exp \left(-t_{n}^{2} / 2 c\right) d t_{n}
$$

where $t_{n}$ is the coordinate function on $R_{n}, n=1,2, \cdots$. Put $X=\prod_{j=1}^{\infty} R_{j}$, $X_{n}=\prod_{j=1}^{n} R_{j}$, and $Y_{n}=\prod_{j=n+1}^{\infty} R_{j} . X, X_{n}$ and $Y_{n}$ are probability spaces. The map $\sum_{j=1}^{n} a_{j} x_{j} \rightarrow \sum_{j=1}^{n} a_{j} t_{j}$ from a dense subset of $H$ into $L^{2}(X)$ is a bounded linear transformation and its extension to $H$ is a representative of the normal distribution on $H$ with parameter $c$. The bounded algebra of $H$ is isomorphic to the bounded algebra of $X$ in a natural way and we concern ourselves henceforth with the probability space $X$.

As $X=X_{n} \times Y_{n}$ we may identify $L^{2}(X)$ with $L^{2}\left(X_{n}\right) \otimes L^{2}\left(Y_{n}\right)$ in the well known way. Since $R_{n}$ and $Y_{n-1}$ are both separable, nonatomic measure spaces of total measure one there exists an isomorphism of the bounded algebra of $Y_{n-1}$ onto the bounded algebra of $R_{n}$ which extends to a unitary transformation, $V_{n}$, of $L^{2}\left(Y_{n-1}\right)$ onto $L^{2}\left(R_{n}\right)$. Let $W_{n}: L^{2}(X) \rightarrow L^{2}\left(X_{n}\right)$ be the unitary transformation defined as follows: write $L^{2}(X)=L^{2}\left(X_{n-1}\right) \otimes L^{2}\left(Y_{n-1}\right)$ and for the range space write $L^{2}\left(X_{n}\right)=L^{2}\left(X_{n-1}\right) \otimes L^{2}\left(R_{n}\right)$. Put $W_{n}=I \otimes V_{n}$ where $I$ 
is the identity operator on $L^{2}\left(X_{n-1}\right) . W_{n}$ restricted to the bounded algebra of $X$ is an isomorphism onto the bounded algebra of $X_{n}$ for it is clearly linear, one to one, onto and on finite linear combinations of functions of the form $f \otimes g$ where $f$ is bounded on $X_{n-1}$ and $g$ is bounded on $Y_{n-1}$, it is multiplicative. Since every element in the bounded algebra of $X$ is an $L^{2}$ limit of a uniformly bounded sequence of elements of this form it follows that $W_{n}$ is an isomorphism.

Now let $U$ be any unitary transformation on $L^{2}(X)$. Put $A_{n}=W_{n} U W_{n}^{-1}$ and $U_{n}=A_{n} \otimes I_{n}$ where $I_{n}$ is the identity on $L^{2}\left(Y_{n}\right) . A_{n}$ and $U_{n}$ are unitary transformations on $L^{2}\left(X_{n}\right)$ and $L^{2}(X)$, respectively. We shall show that $U_{n} \rightarrow U$ strongly as $n \rightarrow \infty$.

Denote by $1^{k}$ the function identically equal to one on $Y_{k}$. Let $g$ be in $L^{2}\left(X_{k}\right)$ and put $f=g \otimes 1^{k}$. Then for $n>k$ we have, putting $1^{\prime}$ for the unit function on $R_{k+1} \times \cdots \times R_{n-1}$ and $1_{n}$ for the unit function on $R_{n}, U_{n} f$ $=U_{n}\left(g \otimes 1^{\prime} \otimes 1_{n} \otimes 1^{n}\right)=\left(W_{n} U W_{n}^{-1}\left(g \otimes 1^{\prime} \otimes 1_{n}\right)\right) \otimes 1^{n}=\left(W_{n} U\left(g \otimes 1^{\prime} \otimes V_{n}^{-1} 1_{n}\right)\right)$ $\otimes 1^{n}=\left(W_{n} U\left(g \otimes 1^{\prime} \otimes 1^{n-1}\right)\right) \otimes 1^{n}=\left(W_{n} U\left(g \otimes 1^{k}\right)\right) \otimes 1^{n}$. Hence $U_{n} f=\left(W_{n} U f\right)$ $\otimes 1^{n}$. As the algebraic span of the subpaces $L^{2}\left(X_{n}\right) \otimes 1^{n}$ is dense in $L^{2}(X)$ there exists for a given number $\epsilon>0$ an integer $N$ and a function $p$ in $L^{2}\left(X_{N}\right)$ such that $h=p \otimes 1^{N}$ satisfies $\|U f-h\|<\epsilon$, where \|\| denotes the norm in $L^{2}(X)$. Now if $n \geqq N+1$ then $\left(W_{n} h\right) \otimes 1^{n}=h$, for writing $h=p \otimes 1^{\prime} \otimes 1^{n-1}$ where $1^{\prime}$ is now the unit on $R_{N+1} \times \cdots \times R_{n-1}$ we have $W_{n} h=p \otimes 1^{\prime} \otimes V_{n} 1^{n-1}$ $=p \otimes 1^{\prime} \otimes 1_{n}$ and $\left(W_{n} h\right) \otimes 1^{n}=p \otimes 1^{\prime} \otimes 1^{n-1}=h$. Hence if $n \geqq \max (N, k)+1$ we have $\left\|U_{n} f-U f\right\|=\left\|\left(W_{n} U f\right) \times 1^{n}-U f\right\|=\left\|\left(W_{n}(U f-h)\right) \times 1^{n}+h-U f\right\|$ $\leqq \|\left(W_{n}(U f-h) \times 1^{n}\|+\| h-U f\|\leqq 2\| U f-h \|<2 \epsilon\right.$. Thus $U_{n} f \rightarrow U f$. As the functions $f$ of the above form are dense in $L^{2}(X)$ it follows from the uniform boundedness of the $U_{n}$ that $U_{n} \rightarrow U$ strongly.

It remains to show that if $U$ is associated with an automorphism of the bounded algebra of $X$ then the $U_{n}$ are also. If $f$ and $g$ are bounded functions on $X_{n}$ then $\left(A_{n} f\right)\left(A_{n} g\right)=\left(W_{n} U W_{n}^{-1} f\right)\left(W_{n} U W_{n}^{-1} g\right)=W_{n}\left(\left(U W_{n}^{-1} f\right)\left(U W_{n}^{-1} g\right)\right)$ $=W_{n}\left(U(1) U\left(\left(W_{n}^{-1} f\right)\left(W_{n}^{-1} g\right)\right)=\left(W_{n} U(1)\right) W_{n} U W_{n}^{-1}(f g)=A_{n}(1) A_{n}(f g)\right.$. Note that we have used the fact that $W_{n}$ extends to an isomorphism from the algebra of all measurable functions on $X$ to the algebra of all measurable functions on $X_{n}$ (modulo null functions). We have also used the symbol, 1 , to denote the unit function on $X$ in the expression $U(1)$ and on $X_{n}$ in the expression $A_{n}(1)$. As $A_{n}(1)>0$ a.e. on $X_{n}, A_{n}$ is associated with an automorphism of the bounded algebra of $X_{n}$. Finally we have, for $f_{i}, g_{i}$ bounded on $X_{n}, \quad Y_{n}$ respectively, $i=1, \quad 2, \quad U_{n}\left(f_{1} \otimes g_{1}\right) \cdot U_{n}\left(f_{2} \otimes g_{2}\right)=\left(\left(A_{n} f_{1}\right)\left(A_{n} f_{2}\right)\right) \otimes g_{1} g_{2}$ $=\left(A_{n}(1) A_{n}\left(f_{1} f_{2}\right)\right) \otimes g_{1} g_{2}=U_{n}(1) U_{n}\left(\left(f_{1} \otimes g_{1}\right)\left(f_{2} \otimes g_{2}\right)\right)$. Thus for finite linear combinations, $h_{1}, h_{2}$ of such decomposable tensors, $f \otimes g$, we have $U_{n}\left(h_{1}\right) U_{n}\left(h_{2}\right)$ $=U_{n}(1) U_{n}\left(h_{1} h_{2}\right)$. Keeping $h_{1}$ fixed and letting $h_{2}$ run through a sequence of uniformly bounded functions in such a way that the functions $h_{2}$ converge in $L^{2}(X)$ while $U_{n}\left(h_{1} h_{2}\right)$ and $U_{n}\left(h_{2}\right)$ converge pointwise almost everywhere on $X$ we see that this equation holds for all bounded measurable functions, $h_{2}$. 
A similar argument shows that the last equation holds for all bounc!ed and measurable functions, $h_{1}$ and $h_{2}$, on $X$ and completes the proof of the theorem.

REMARK. It is straightforward to verify that the group of those unitaries on $L^{2}(H)$ which are associated with automorphisms, i.e., which satisfy conditions (A) is closed in the strong operator topology.

CoRollary 3.1. Let II be a real Hilbert space of arbitrary dimension. I?note by $G$ the group of unitaries on $L^{2}(H)$ which are associatcd with automorphisms of the bounded algebra of $H$ and by $G_{0}$ the subgroup of $G$ consisting of those unitaries in $G$ which are based on finite dimensional subspaces of $I I$. Then $G_{0}$ is dense in $G$ in the strong opcrator topology.

Proof. The corollary follows immediately from Theorem 3 in case $I I$ is separable so we consider an inseparable space, $I I$, and a unitary transformation, $U$, on $L^{2}(H)$ which is associated with an automorphism of the bounded algebra of $H$.

Since the bounded measurable tame functions on $H$ are dense in $L^{2}(I I)$ every function in $L^{2}(H)$ is based on a separable subspace of $H$. Furthermore, if $M$ is a separable subspace of $L^{2}(H)$ then there exists a separable subspace, $K$, of $H$ such that every vector in $M$ is based on $K$. For if $f_{1}, f_{2}, \ldots$ is an orthonormal basis of $M$ and $K_{n}$ is a separable subspace of $H$ on which $f_{n}$ is based, $n=1,2, \cdots$ we may take $K=\operatorname{span}\left\{K_{n}: n=1,2, \cdots\right\}$. Indeed $L^{2}(K) \otimes 1$ is a closed subspace of $L^{2}(H)$ where 1 is the function identically equal to one on $K^{\perp}$ and as each $f_{n}$ is in $L^{2}(K) \otimes 1, M$ is also contained in $L^{2}(K) \otimes 1$.

Now suppose that $g_{1}, \cdots, g_{k}$ are in $L^{2}(H)$. Let $H_{1}$ be a separable subspace of $H$ on which $g_{1}, \cdots, g_{k}$ are all based. For any subspace $K$ of $H$ we denote by $B(K)$ the subspace of $L^{2}(H)$ consisting of elements based on $K$, i.e., $B(K)=L^{2}(K) \otimes 1$ where 1 denotes here the unit function on $K^{\perp}$. We define an increasing sequence of separable subspaces, $H_{n}$, of $H$ inductively by observing that $U B\left(H_{n}\right)$ and $U^{*} B\left(H_{n}\right)$ are both separable when $H_{n}$ is so that there exists a separable subspace, $H_{n+1}$, of $H$ with the property that $B\left(H_{n+1}\right)$ contains $\operatorname{span}\left(B\left(H_{n}\right), U B\left(H_{n}\right), U^{*} B\left(H_{n}\right)\right)$. Then $H_{n+1}$ contains $H_{n}$ and $K=\operatorname{span}\left\{H_{n}: n=1,2, \cdots\right\}$ is a separable subspace of $H . B(K)$ is invariant under $U$ and $U^{*}$ for it is clear that the algebraic linear span of the subspaces $B\left(H_{n}\right)$ is invariant under $U$ and $U^{*}$ so that it suffices to prove that the algebraic linear span of the $B\left(H_{n}\right)$ is dense in $B(K)$. To this end let $x_{1}, x_{2}, \cdots$ be an orthonormal basis of $K$ such that all $x_{j}$ are in the algebraic linear span of the $H_{n}$. Functions of the form $f(x)=\phi\left(\left(x, x_{1}\right), \cdots,\left(x, x_{m}\right)\right)$ where $\phi$ is a bounded Baire function on $E_{m}$ are dense in $L^{2}(K)$ and also are in the algebraic linear span of the $B\left(H_{n}\right)$. We note that $g_{1}, \cdots, g_{k}$ are in $B(K)$.

Now the map $f \rightarrow f \otimes 1$ where 1 is the unit function on $K^{\perp}$ is a unitary transformation from $L^{2}(K)$ onto $B(K)$ which is an algebraic isomorphism from the bounded algebra of $K$ into the bounded algebra of $H$. Thus the restriction 
of $U$ to $B(K)$ gives rise to a unitary transformation of $L^{2}(K)$ which is associated with an automorphism of the bounded algebra of $K$. Theorem 3 now applies and for a given $\epsilon>0$ ensures the existence of a unitary transformation, $V$, on $L^{2}(K)$ which is associated with an automorphism of the bounded algebra of $K$, is based on a finite dimensional subspace of $K$ and saiisfies $\left\|(U-V) g_{i}\right\|<\epsilon, i=1, \cdots, k$ wherein some obvious identifications have been made. Putting $W=V \otimes I$ in the decomposition $L^{2}(H)=L^{2}(K) \otimes L^{2}\left(K^{\perp}\right)$ the corollary follows.

3. Absolute continuity of distributions. Before proceeding to the main result of this section we shall discuss weak distributions in more detail. Let $m$ be a representative of a weak distribution on a real linear topological space, $L$. Then $m$ is a linear map from $L^{*}$ to measurable functions on a probability space which we shall denote by $(S, \delta, \mu)$ where $\mu$ is a probability measure on a $\sigma$-algebra, $\mathcal{S}$, of subsets of $S$. Denote by $\delta_{0}$ the smallest $\sigma$-algebra of subsets of $S$ with respect to which all the measurable functions, $m(y), y \in L^{*}$, are measurable. Then $S_{0} \subset \mathcal{S}$ and $\mu$ is defined on $S_{0}$. Let $\mathfrak{N}$ be the algebra of bounded complex valued $S_{0}$-measurable functions on $S$ modulo the ideal of null functions and, identifying an element of $\mathfrak{T}$ with a function on $S$ in the customary way, put $E_{m}(f)=\int_{S} f d \mu$ for $f$ in $\mathfrak{T}$. If $m^{\prime}$ is another representative of the same weak distribution on $L$ with values on $\left(S^{\prime}, \boldsymbol{s}^{\prime}, \mu^{\prime}\right)$ and if $\mathfrak{M}^{\prime}$ denotes the similarly defined algebra then it is known [14] that there exists a unique isomorphism from $\mathfrak{T}$ onto $\mathfrak{T}^{\prime}$ which preserves expectation and whose extension, $\phi$, to the measurable functions on $\left(S, \varsigma_{0}, \mu\right)$ takes $m(y)$ into $m^{\prime}(y)$ for all $y$ in $L^{*}$. The weak distribution therefore defines a unique (up to an expectation preserving natural isomorphism) algebra, $\mathfrak{T}$, with expectation functional, $E$, which we shall call the bounded algebra of $L$ with respect to the weak distribution. The $L^{p}$ space of $L$ with respect to the weak distribution may be defined as the completion of $\mathfrak{T}$ with respect to the norm $\|f\|$ $=(E(|f| p))^{1 / p}$ and may be identified with $L^{p}\left(S, S_{0}, \mu\right)$. Convergence in probability likewise has an invariant meaning in that a sequence of functions $\left\{f_{n}\right\}$ which are measurable on $\left(S, s_{0}, \mu\right)$ and which converge in probability to $f$ correspond under $\phi$ to a sequence, $f_{n}^{\prime}=\phi\left(f_{n}\right)$, of measurable functions on $\left(S^{\prime}, S_{0}^{\prime}, \mu^{\prime}\right)$ which converge in probability to $\phi(f)$. As in the case of the normal distribution on a Hilbert space we shall refer to a measurable function on $\left(S, \delta_{0}, \mu\right)$ (mod null functions) as a measurable function on $L$ with respect to the given weak distribution. We shall continue to be concerned with a real Hilbert space, $H$. It may be well to point out that if $f(x)=v\left(\left(x, y_{1}\right), \cdots,\left(x, y_{k}\right)\right)$ is a tame function on $H, v\left(t_{1}, \cdots, t_{k}\right)$ being a Baire function on $E_{k}$, then the corresponding measurable function with respect to the weak distribution with respresentative, $m$, is $v\left(m\left(y_{1}\right), \cdots, m\left(y_{k}\right)\right)$.

The following definition is due to Segal [16].

Definition 4 . Let $m$ and $n$ be representatives of two weak distributions on a real topological linear space, $L$. The distribution represented by $m$ is 
absolutely continuous with respect to the distribution represented by $n$ if there exists a complete homomorphism, $\phi$, from the bounded algebra, $\mathscr{N}$, of $L$ with respect to $n$ into the bounded algebra, $\mathfrak{T}$, of $L$ with respect to $m$ whose extension to the algebra of measurable functions on $L$ with respect to $n$ takes $n(y)$ into $m(y)$ for all $y$ in $L^{*}$. Herein a complete homomorphism is a homomorphism which preserves complex conjugation and is completely additive on orthogonal families of projections, i.e. $\phi\left(\sum_{\alpha} P_{\alpha}\right)=\sum_{\alpha} \phi\left(P_{\alpha}\right)$ whenever $\left\{P_{\alpha}\right\}$ is an orthogonal family of projections in $\Re$. An infinite sum is to be interpreted as convergence in probability. The derivative of $m$ with respect to $n$, denoted by $d m / d n$, is defined as the unique function in $L^{1}(L, n)$ such that $E_{m}(\phi(f))=E_{n}((d m / d n) f)$ for all $f$ in $\mathfrak{N}$ where $E_{m}$ and $E_{n}$ are the expectation functionals.

Two distributions are equivalent in the sense of absolute continuity if each is absolutely continuous with respect to the other.

REMARKs. It is readily seen that absolute continuity is a property of the distributions and not merely of their representatives.

When $L$ is finite dimensional the distributions $m$ and $n$ give rise to measures $\mu$ and $\nu$, respectively, on $L$ as in [14] and absolute continuity of $m$ with respect to $n$ is equivalent to absolute continuity of $\mu$ with respect to $\nu$.

In the following we shall say that an operator, $A$, on a Hilbert space is of trace class if $\left(A^{*} A\right)^{1 / 2}$ has a convergent trace on every orthonormal basis. As is known [12] the operators of trace class form a Banach space under the norm $\|A\|_{1}=$ trace $\left(\left(A^{*} A\right)^{1 / 2}\right)$.

If $T$ is a (not necessarily linear) transformation of $H$ into itself a bounded linear operator, $A$, will be called the Frechet derivative of $T$ at the point, $x$, in $H$ if $\lim _{y \rightarrow 0}\|T(x+y)-T(x)-A y\| /\|y\|=0$. We shall denote the Frechet derivative of $T$ at $x$ by $T_{x}$.

Theorem 4. Let $K$ be a transformation of a real Hilbert space, $H$, into itself such that $K(0)=0$. Assume that $K$ possesses a Frechet derivative, $K_{x}$, of trace class at each point, $x$, in $H$ and that $K_{x}$ has the following properties:

(1) $\left\|K_{x}\right\| \leqq \lambda<1$ for all $x$ in $H$ and some real number, $\lambda$.

(2) The map $x \rightarrow K_{x}$ is uniformly continuous near zero in the topology $\mathrm{H}_{2}$ with the trace class norm topology on the range.

(3) There exist Hilbert-Schmidt operators, B, C, such that $\left|\left(K_{x} y, z\right)\right|$ $\leqq\|C y\|\|B z\|$ for all $x, y, z$ in $H$.

Let $T=I+K$ where $I$ is the identity operator on $H$. Then for each $y$ in $H$ the function $(T(\cdot), y)$ is uniformly continuous in the topology $\mathrm{H}_{2}$ and defines a measurable function, $m(y)$, on $H$ with respect to the normal distribution. The map $y \rightarrow m(y)$ defines a distribution on $H$ which is equivalent to the normal distribution. The function

$$
g(x)=\exp \left[\left(-2(K x, x)-\|K x\|^{2}\right) / 2 c\right] \operatorname{det}\left[\left(T_{x}{ }^{*} T_{x}\right)^{1 / 2}\right]
$$

is uniformly continuous near zero in the topology $\mathrm{H}_{2}$. If $\tilde{g}$ denotes the correspond- 
ing measurable function on $H$ with respect to the normal distribution and if $u$ is a bounded complex valued Baire function on $E_{k}$ then

$$
E\left(u\left(m\left(y_{1}\right), \cdots, m\left(y_{k}\right)\right) \tilde{g}\right)=E\left(u\left(n\left(y_{1}\right), \cdots, n\left(y_{k}\right)\right)\right)
$$

where $y_{1}, \cdots, y_{k}$ are arbitrary vectors in $H$ and $E$ is the expectation functional of the normal distribution.

The proof depends on the following lemmas. As there is no loss in generality we shall assume that $B$ and $C$ are non-negative (symmetric) operators.

LEMma 4.1. Let $S$ be the metric space consisting of those trace class operators $B$ on $H$ such that $I+B$ is nonsingular, i.e., has a bounded inverse, with metric $d(A, B)=\|A-B\|_{1}$. The determinant function

$$
\Delta(B)=\operatorname{det}|I+B|=\exp \left[\frac{1}{2} \operatorname{trace}\left(\log (I+B)^{*}(I+B)\right)\right]
$$

is a continuous function of $B$ on $\mathcal{S}$. If $R$ is a subset of $\mathcal{S}$ such that $\|B\|_{1}$ and $\left\|(I+B)^{-1}\right\|$ are bounded for $B$ in $R$ then $\Delta(B)$ is uniformly continuous on $R$ and is bounded and bounded away from zero on $R$.

Proof. If $I+C=(I+B)^{*}(I+B)$. Then $I+C$ is nonsingular when $B$ is in $\mathcal{S}$ and $C=B+B^{*}+B^{*} B$ is then also in $\delta$. Using the facts that $\|B\| \leqq\|B\|_{1}$ and $\|A B\|_{1} \leqq\|A\|\|B\|_{1}$ whenever $A$ is bounded it is easy to see that the map $B \rightarrow C$ is continuous on $S$ and uniformly so on bounded subsets. For each $\alpha$ such that $0<\alpha<1$ let $Q_{\alpha}$ be the set of those symmetric operators $C$ in $S$ such that $((I+C) x, x)>\alpha$ for all unit vectors $x$. Let $Q=U_{0<\alpha<1} Q_{\alpha}$. Then each $a_{\alpha}$ is open in $Q$ for if $C_{0}$ is in $Q_{\alpha}$ then since $C_{0}$ is completely continuous the greatest lower bound of the spectrum of $C_{0}$ is an eigenvalue of $C_{0}$ hence there exists $\epsilon>0$ such that $\left(\left(I+C_{0}\right) x, x\right)>\alpha+\epsilon$ whenever $\|x\|=1$. Hence if $C$ is in $Q$ and $\left\|C-C_{0}\right\|_{1}<\epsilon$ then $\left\|C-C_{0}\right\|<\epsilon$ and $((I+C) x, x)>\alpha$ for all unit vectors $x$. It is readily verified that, for some $\alpha>0,(I+B)^{*}(I+B)-I$ is in $a_{\alpha}$ for all $B$ in $R$. Since the exponential function is uniformly continuous on bounded intervals and the trace function is uniformly continuous on the Banach space of trace class operators it suffices to show that the map $C \rightarrow \log (I+C)$ is continuous on each $a_{\alpha}$ with the trace class norm on the range and is uniformly continuous on bounded subsets of each $a_{\alpha}$ with bounded range on such subsets.

If $C$ is in $Q_{\alpha}$ then by the spectral theorem and the relation $\log (1+x)$ $=x \int_{0}^{1}(1+t x)^{-1} d t$ we have $\log (I+C)=C \int_{0}^{1}(I+t C)^{-1} d t$. Hence $\|\log (I+C)\|_{1}$ $\leqq\|C\|_{1} \int_{0}^{1}\left\|(I+t C)^{-1}\right\| d t \leqq \alpha^{-1}\|C\|_{1}$. Hence $\log (I+C)$ is of trace class and it follows further that $\Delta(B)$ is bounded and bounded away from zero for $B$ in R. Furthermore,

$$
\log (I+C)-\log \left(I+C_{0}\right)=\int_{0}^{1}\left[C(I+t C)^{-1}-C_{0}\left(I+t C_{0}\right)^{-1}\right] d t
$$


Starting with the identity

$$
\left(I+t C_{0}\right)^{-1}\left(I+t C_{0}\right)+t\left(C-C_{0}\right)(I+t C)^{-1}=\left(I+t C_{0}\right)^{-1}
$$

we obtain

$$
(I+t C)^{-1}+t\left(I+t C_{0}\right)^{-1}\left(C-C_{0}\right)(I+t C)^{-1}=\left(I+t C_{0}\right)^{-1} .
$$

Multiplying by $C$ on the left and then subtracting $C_{0}\left(I+t C_{0}\right)^{-1}$ we get $C(I+t C)^{-1}-C_{0}\left(I+t C_{0}\right)^{-1}$

$$
=\left(C-C_{0}\right)\left(I+t C_{0}\right)^{-1}-t\left(I+t C_{0}\right)^{-1}\left(C-C_{0}\right)(I+t C)^{-1} .
$$

It follows that if $C_{0}$ and $C$ are in $Q_{\alpha}$ then $\left\|\log (I+C)-\log \left(I+C_{0}\right)\right\|_{1}$ $\leqq \alpha^{-1}\left\|C-C_{0}\right\|_{1}+\alpha^{-2}\left\|C-C_{0}\right\|_{1}$. This completes the proof of the lemma.

Lemma 4.2. Let $A, B, C$ be bounded linear operators on a Hilbert space $H$ such that $B^{*}=B, C^{*}=C$ and for all $x, y$ in $H|(A x, y)| \leqq\|C x\|\|B y\|$. Then there exists a unique operator $D$ on $H$ such that (1) $\|D\| \leqq 1,(2) A=B D C$, (3) $R(D) \subset N(B)^{\perp}$ (4) $N(D) \supset R(C)^{\perp}$ where $R(E)$ and $N(E)$ denote the range and nullspace, respectively, of an operator $E$.

Proof. Define $E_{0}$ on $R(C)$ by $E_{0} C x=A x . E_{0}$ is well defined for if $C x=0$ then $(A x, y)=0$ for all $y$ in $H$, hence $A x=0$. Further, $\left\|E_{0} C x\right\|$ $=\sup _{y}\left\|_{=1}\left|\left(E_{0} C x, y\right)\right|=\sup _{y}\right\|_{=1}|(A x, y)| \leqq\|C x\|\|B\|$. Hence $\left\|E_{0}\right\| \leqq\|B\|$. $E_{0}$ may be extended to a bounded linear operator on $\mathrm{Cl} R(C)(=R(C)$ closure) which may be further extended linearly to an operator $E$ on all of $H$ by defining $E$ to be zero on $R(C)^{\perp}$. Thus $\|E\| \leqq\|B\|, N(E) \supset R(C)^{\perp}$ and $A=E C$. It follows that $\mathrm{Cl} R\left(E^{*}\right) \subset \mathrm{Cl} R(C)$ and $\left|\left(C x, E^{*} y\right)\right| \leqq\|C x\|\|B y\|$. Hence $\left\|E^{*} y\right\| \leqq\|B y\|$. Define $F_{0}$ on $R(B)$ by $F_{0} B y=E^{*} y$. $F_{0}$ is clearly well defined and $\left\|F_{0}\right\| \leqq 1$. Upon closing $F_{0}$ and extending its domain to all of $H$ by making it zero on $R(B)^{\perp}$ we obtain a linear operator $F$ such that $\|F\| \leqq 1, F B=E^{*}$ and $N(F) \supset R(B)^{\perp}$. Put $D=F^{*}$. Then $A=E C=B F^{*} C=B D C,\|D\| \leqq 1$, $\mathrm{Cl} R(D)=N(F)^{\perp} \mathrm{CCl} R(B)=N(B)^{\perp}$ and $N(D)=R(F)^{\perp}=R\left(E^{*}\right)^{\perp}=N(E)$ $\supset R(C)^{\perp}$.

If $D^{\prime}$ is another bounded operator satisfying (2), (3) and (4) then $D^{\prime \prime}$ $=D-D^{\prime}$ satisfies (3), (4) and $B D^{\prime \prime} C=0$ which together imply $D^{\prime \prime}=0$ completing the proof of the lemma.

Remark. If $K$ is a map of $H$ into itself possessing a Frechet derivative, $K_{x}$, which is a strongly continuous function of $x$ then

$$
K(x)-K(y)=\int_{0}^{1} K_{t(x-y)+y}(x-y) d t
$$

where the integral may be taken in the Riemann sense. This may be proved by observing that for arbitrary $z$ in $H$ the functions

$$
f(s)=(K(s(x-y)+y)-K(y), z)
$$


and $g(s)=\int_{0}^{s}\left(K_{t(x-y)+y}(x-y), z\right) d t$ agree at $s=0$ and have the same derivative for all $s$ so that by the fundamental theorem of calculus $f(1)=g(1)$.

Lemma 4.3. Let $K$ be a transformation of a real Hilbert space $H$ into itself which possesses a Frechet derivative $K_{x}$ at each point $x$ of $H$. Assume that the map $x \rightarrow K_{x}$ is continuous on $H$ with the strong operator topology on the range and that $K_{x}$ satisfies condition (3) of the theorem. Put $T=I+K$. Then for each $y$ in $H$ the function $x \rightarrow(T x, y)$ is uniformly continuous in the topology $\mathrm{H}_{2}$ and determines a measurable function $m(y)$ on $H$ with respect to the normal distribution. The map $y \rightarrow m(y)$ is a distribution on $H$.

Proof. $(x, y)$ is a uniformly continuous function of $x$ in the weak topology on $H$ hence also in the stronger topology $H_{2}$. Applying Lemma 4.2 to the operators $K_{x}$ it follows that there exists an operator valued function $D_{x}$, $x \in H$, such that $\left\|D_{x}\right\| \leqq 1$ and $K_{x}=B D_{x} C$ for all $x$ in $H$ where $B$ and $C$ are symmetric Hilbert-Schmidt operators. By the preceding remark we have $(K(x)-K(z), y)=\int_{0}^{1}\left(K_{t(x-z)+z}(x-z), y\right) d t$. Hence

$$
|(K(x)-K(z), y)| \leqq\|B y\|\|C(x-z)\| \text {. }
$$

Thus $(K(\cdot), y)$ is also uniformly continuous in $H_{2}$ and the measurable function $m(y)=\lim _{\text {in }}$ probability ${ }_{P \rightarrow I}(T P(\cdot), y)$ exists by Theorem 1 . Linearity of $m(\cdot)$ is obvious.

REMARK. We note that the net $((I+P K P)(\cdot), y)$ also converges to $m(y)$ in probability as $P \rightarrow I$ through the directed set of finite dimensional projections since the terms of this net coincide with those of $(T P(\cdot), y)$ for those $P$ for which $P y=y$.

Lemma 4.4. Assume hypothesis of Lemma 4.3 and assume in addition that $K(0)=0$. The function $f(x)=(K x, x)$ is uniformly continuous near zero in the topology $H_{2}$. The net $f(P x)=(P K P x, x)$ of measurable tame functions converges in probability as $P \rightarrow I$ through the directed set of finite dimensional projections on $H$. If $A=\left(B^{2}+C^{2}\right)^{1 / 2}$ then $|(K x, x)| \leqq\|A x\|^{2}$.

Proof. Using equation (2) we may write $|f(x)-f(y)| \leqq|(K x, x-y)|$ $+|(K x-K y, y)| \leqq\|C x\|\|B(x-y)\|+\|B y\|\|C(x-y)\|$. Hence if $\|A x\|<n$ and $\|A y\|<n$ and $\|A(x-y)\|<\epsilon / 2 n$ then $|f(x)-f(y)|<\epsilon$. Hence $f$ is uniformly continuous in $H_{2}$ on each of the sets $\left\{x:\left\|A_{n} x\right\|<1\right\}$ where $A_{n}=n^{-1} A$. Further $|(K x, x)| \leqq\|C x\|\|B x\| \leqq\|A x\|^{2}$. The remainder of the lemma follows from Theorem 1.

Lemma 4.5. Assume hypothesis of Lemma 4.4. Then the function $h(x)$ $=\|K x\|^{2}$ is uniformly continuous near zero in the topology $H_{2}$. Let $\tilde{h}$ denote the corresponding measurable function on $H$ with respect to the normal distribution. The net $\|P K P x\|^{2}$ of measurable tame functions converges in probability to $\tilde{h}$ as $P \rightarrow I$ through the directed set of finite dimensional projections on $H$. 
Proof. It follows from equation (2) that $\|K x-K y\| \leqq\|B\|\|C(x-y)\|$. Hence

$$
\begin{aligned}
\left|\|K x\|^{2}-\|K y\|^{2}\right| & \leqq(\|K x\|+\|K y\|)|\|K x\|-\|K y\|| \\
& \leqq\|B\|^{2}(\|C x\|+\|C y\|)\|C(x-y)\|
\end{aligned}
$$

showing that $\|K x\|^{2}$ is uniformly continuous in $H_{2}$ on each of the sets $\{x:\|C x\|<n\}$ and is therefore uniformly continuous near zero in $H_{2}$. It is also clear that the function $\|K x\|$ is uniformly continuous in $H_{2}$. Letting $f(x)=\|K x\|$ and denoting by $\tilde{f}$ the corresponding measurable function the lemma will follow if we show that $\lim$ in probability ${ }_{P \rightarrow I}\|P K P(\cdot)\|=\tilde{f}$. Now $\|K P x\|-\|P K P x\| \mid \leqq\|(I-P) K P x\| \leqq \int_{0}^{1}\left\|(I-P) B D_{t P x} C P x\right\| d t$ $\leqq\|(I-P) B\|\|C P x\|$ which converges to zero in probability as $P \rightarrow I$ since $\|(I-P) B\| \rightarrow 0$ uniformly while $\|C P x\|$ converges in probability by Lemma 1.2. As $|\tilde{f}-\|P K P(\cdot)\|| \leqq|\tilde{f}-h \circ P|+|\|K P(\cdot)\|-\|P K P(\cdot)\||$ and as both terms on the right converge to zero in probability as $P \rightarrow I$ the proof is complete.

LEMMA 4.6. Let $K$ satisfy the hypothsis of Lemma 4.4 and assume also that $K_{x}$ satisfies condition (1) of the theorem. For each projection $P$ in the set $\mathcal{F}$ of finite dimensional projections on $H$ put $T_{P}=I+P K P$. Let $A=\left(B^{2}+C^{2}\right)^{1 / 2}$. Then $\left(T_{P}\right)^{-1}$ exist for each $P$ and the net $\left\|A P\left(T_{P}\right)^{-1} x\right\|$ of measurable tame functions converges in probability as $P \rightarrow I$ in the directed set $\mathcal{F}$.

Proof. Put $K_{P}=P K P$. It follows from the remark preceding Lemma 4.3 and from $\left\|\left(K_{P}\right)_{x}\right\|=\left\|P K_{P x} P\right\| \leqq\left\|K_{P x}\right\| \leqq \lambda<1$ that $\left\|K_{P} x-K_{P} y\right\| \leqq \lambda\|x-y\|$ which implies that $T_{P}$ is one to one for if $T_{P} x=T_{P} y$ then $x-y=K_{P} y-K_{P} x$ and $\|x-y\| \leqq \lambda\|x-y\|$ so that $x=y$. That $T_{P}$ is onto follows from a well known successive approximations construction of $\left(T_{P}\right)^{-1}$ which construction we shall carry out here as the approximations are needed in the remainder of the proof. Let $\left(S_{P}\right)_{0}=I$ and define inductively $\left(S_{P}\right)_{n}=I-K_{P}\left(S_{P}\right)_{n-1}$. Then $\left\|\left[\left(S_{P}\right)_{n}-\left(S_{P}\right)_{n-1}\right] x\right\|=\left\|\left(K_{P}\left(S_{P}\right)_{n-1}-K_{P}\left(S_{P}\right)_{n-2}\right) x\right\| \leqq \lambda\left\|\left[\left(S_{P}\right)_{n-1}-\left(S_{P}\right)_{n-2}\right] x\right\|$. Thus by induction we have $\left\|\left[\left(S_{P}\right)_{n}-\left(S_{P}\right)_{n-1}\right] x\right\| \leqq \lambda^{n-1}\left\|K_{P} x\right\|$. As $\| K_{P} x$ $=\left\|P \int_{0}^{1} B D_{t P x} C P x d t\right\| \leqq\|B\|\|C P x\|$ it follows that $\left\|\left[\left(S_{P}\right)_{n+k}-\left(S_{P}\right)_{n}\right] x\right\|$ $\leqq \sum_{j=1}^{k}\left\|\left[\left(S_{P}\right)_{n+j}-\left(S_{P}\right)_{n+j-1}\right] x\right\| \leqq \sum_{j=1}^{k} \lambda^{n+j-1}\|B\|\|C P x\|$. Thus the sequence $\left(S_{P}\right)_{n} x$ converges uniformly on the set $\{x:\|C P x\|<M\}$ for each real number $M$. Let $S_{P} x=$ strong limit ${ }_{n \rightarrow \infty}\left(S_{P}\right)_{n} x$. As $T_{P}$ is continuous in the norm topology of $H$ we have $T_{P} S_{P} x=\lim _{n \rightarrow \infty} T_{P}\left(S_{P}\right)_{n} x=\lim _{n \rightarrow \infty}\left(I+K_{P}\right)\left(S_{P}\right)_{n} x$ $=\lim _{n \rightarrow \infty}\left[\left(S_{P}\right)_{n} x+x-\left(S_{P}\right)_{n-1} x\right]=x$. Hence $T_{P}$ is onto and $S_{P}$ is its (left and right) inverse.

Now we show by induction that for each integer $n$ the measurable tame functions $\left\|A\left(P\left(S_{P}\right)_{n}-Q\left(S_{Q}\right)_{n}\right) x\right\|$ and $\left\|A\left(\left(S_{P}\right)_{n}-\left(S_{Q}\right)_{n}\right) x\right\|$ converge to zero in probability as $P$ and $Q$ approach $I$ through $\mathcal{F}$. That these are actually tame functions follows from the fact that $P$ commutes with $\left(S_{P}\right)_{n}$ (which can be established easily by induction on $n)$ and from the fact that $P\left(\left(S_{P}\right)_{n}-I\right)$ 
$=-P K_{P}\left(S_{P}\right)_{n-1}=-K_{P}\left(S_{P}\right)_{n-1}=\left(S_{P}\right)_{n}-I$ for then $\left(S_{P}\right)_{n}-\left(S_{Q}\right)_{n}$ $=\left(\left(S_{P}\right)_{n}-I\right) P-\left(\left(S_{Q}\right)_{n}-I\right) Q$ so that $\left\|A\left(\left(S_{P}\right)_{n}-\left(S_{Q}\right)_{n}\right) x\right\|$ is also a tame function. Now observe that

$$
\begin{aligned}
\left\|A\left(P\left(S_{P}\right)_{n}-Q\left(S_{Q}\right)_{n}\right) x\right\| & \left.=\| A\left[P+P\left(\left(S_{P}\right)_{n}-I\right)-Q-Q\left(S_{Q}\right)_{n}-I\right)\right] x \| \\
& \leqq\|A(P-Q) x\|+\left\|A\left(\left(S_{P}\right)_{n}-\left(S_{Q}\right)_{n}\right) x\right\| .
\end{aligned}
$$

Since the first term in the last line converges to zero in probability by Lemma 1.2 as $P$ and $Q$ approach $I$ through $\mathcal{F}$ it suffices to show that, for each integer $n,\left\|A\left(\left(S_{P}\right)_{n}-\left(S_{Q}\right)_{n}\right) x\right\|$ converges to zero in probability under the induction assumption that $\left\|A\left(P\left(S_{P}\right)_{k}-Q\left(S_{Q}\right)_{k}\right) x\right\|$ and $\left\|A\left(\left(S_{P}\right)_{k}-\left(S_{Q}\right)_{k}\right) x\right\|$ converge to zero in probability for each $k<n$ as $P$ and $Q$ approach $I$ through $\mathcal{F}$. Note that for $k=0$ the assumption is true. Equation (2) implies $\|K x-K y\|$ $\leqq\|B\|\|C(x-y)\|$. Hence $\|K x-K y\| \leqq\|B\|\|A(x-y)\|$. Thus

$$
\begin{aligned}
& \left\|A\left[\left(S_{P}\right)_{n}-\left(S_{Q}\right)_{n}\right] x\right\|=\left\|A\left[Q K Q\left(S_{Q}\right)_{n-1}-P K P\left(S_{P}\right)_{n-1}\right] x\right\| \\
& \quad=\| A\left[(Q-P) K Q\left(S_{Q}\right)_{n-1}+P\left(K Q\left(S_{Q}\right)_{n-1}-K P\left(S_{P}\right)_{n-1}\right] x \|\right. \\
& \quad \leqq\|A(Q-P)\|\|B\|\left\|A Q\left(S_{Q}\right)_{n-1} x\right\|+\|A\|\|B\|\left\|A\left[Q\left(S_{Q}\right)_{n-1}-P\left(S_{P}\right)_{n-1}\right] x\right\| .
\end{aligned}
$$

The first term in the last line converges to zero in probability since $\|A(Q-P)\|$ converges to zero uniformly as $P$ and $Q$ approach $I$ while $\left\|A Q\left(S_{Q}\right)_{n-1} x\right\|$ converges in probability as a consequence of the induction assumption. The second term on the right also converges to zero by the induction assumption. This proves the assertion of this paragraph.

As $P$ commutes with $\left(S_{P}\right)_{n}$ for each $n$ it also commutes with their strong limit, $S_{P}$, and similarly we have $\left(S_{P}-I\right) P=P\left(S_{P}-I\right)=S_{P}-I$. As $S_{P}$ is a strong limit of continuous transformations which converge uniformly on the sets $\{x:\|C P x\|<M\}$ and in particular on bounded sets $S_{P}$ is continuous so $\left\|A P S_{P} x\right\|$ is a measurable tame function. As

$$
\begin{aligned}
\left|\left\|A P S_{P} x\right\|-\left\|A Q S_{Q} x\right\|\right| & \leqq\left\|A P S_{P} x-A Q S_{Q} x\right\| \\
& \leqq\|A(P-Q) x\|+\left\|A\left(S_{P}-S_{Q}\right) x\right\|
\end{aligned}
$$

and as the first term in the last line converges to zero in probability it suffices for the proof of the lemma to show that the tame functions $\left\|A\left(S_{P}-S_{Q}\right) x\right\|$ converge to zero in probability as $P$ and $Q$ approach $I$ through $\mathcal{F}$.

Let $\epsilon$ and $\delta$ be positive real numbers. Since $\|C P x\|$ converges in probability as $P \rightarrow I$ by Lemma 1.2 there exists a number $M$ and a projection $P_{0}$ in $\mathfrak{F}$ such that $P \geqq P_{0}$ implies $\operatorname{Prob}(\|C P x\| \geqq M)<\delta / 3$. There exists an integer $N$ such that $\left\|A\left(S_{P}-\left(S_{P}\right)_{N}\right) x\right\|<\epsilon / 3$ whenever $\|C P x\|<M$. Note that $N$ is independent of $P$. Thus

$$
\operatorname{Prob}\left(\left\|A\left(S_{P}-\left(S_{P}\right)_{N}\right) x\right\| \geqq \epsilon / 3\right)<\delta / 3 \text { whenever } \mathrm{P} \geqq P_{0} .
$$

Since $\left\|A\left(\left(S_{P}\right)_{N}-\left(S_{Q}\right)_{N}\right) x\right\|$ converges to zero in probability as $P$ and $Q$ approach $I$ through $\mathcal{F}$ there exists a projection $P_{1} \geqq P_{0}$ in $\mathcal{F}$ such that if $P$ and $Q$ 
are in $\mathcal{F}$ and $P \geqq P_{1}$ and $Q \geqq P_{1}$ then

$$
\operatorname{Prob}\left(\left\|A\left(\left(S_{P}\right)_{N}-\left(S_{Q}\right)_{N}\right) x\right\| \geqq \epsilon / 3\right)<\delta / 3 .
$$

In view of the inequality

$$
\begin{aligned}
& \left\|A\left(S_{P}-S_{Q}\right) x\right\| \leqq\left\|A\left(S_{P}-\left(S_{P}\right)_{N}\right) x\right\| \\
& \quad+\left\|A\left(\left(S_{P}\right)_{N}-\left(S_{Q}\right)_{N}\right) x\right\|+\left\|A\left(\left(S_{Q}\right)_{N}-S_{Q}\right) x\right\|
\end{aligned}
$$

the left hand side is greater or equal to $\epsilon$ only if at least one term on the right is greater or equal to $\epsilon / 3$. Hence if $P$ and $Q$ are in $\mathcal{F}$ and $P \geqq P_{1}$ and $Q \geqq P_{1}$ then

$$
\begin{aligned}
\operatorname{Prob}\left(\left\|A\left(S_{P}-S_{Q}\right) x\right\| \geqq \epsilon\right) \leqq & \operatorname{Prob}\left(\left\|A\left(S_{p}-\left(S_{P}\right)_{N}\right) x\right\| \geqq \epsilon / 3\right) \\
& +\operatorname{Prob}\left(\left\|A\left(\left(S_{P}\right)_{N}-\left(S_{Q}\right)_{N}\right) x\right\| \geqq \epsilon / 3\right) \\
& +\operatorname{Prob}\left(\left\|A\left(\left(S_{Q}\right)_{N}-S_{Q}\right) x\right\| \geqq \epsilon / 3\right) \\
< & 3(\delta / 3)=\delta .
\end{aligned}
$$

This completes the proof of the lemma.

Lemma 4.7. Let $K$ satisfy the hypothesis of the theorem and put $K_{P}=P K P$ for each finite dimensional projection $P$. The functions $\Delta\left(\left(K_{\Gamma}\right)_{x}\right)$ $=\left|\operatorname{det}\left(I+\left(K_{P}\right)_{x}\right)\right|$ are tame functions and the corresponding net of measurable tame functions converges in probability as $P \rightarrow I$ in the directed set $\mathcal{F}$ of finite dimensional projections. There exist real numbers $a$ and $b$ such that $0<a$ $\leqq \Delta\left(\left(K_{P}\right)_{x}\right) \leqq b$ for all $x$ and $P$ in $\mathfrak{F}$.

Proof. As $\left(K_{P}\right)_{x}=P K_{P x} P$ it follows that $\Delta\left(\left(K_{P}\right)_{x}\right)$ is a continuous tame function, that $\left\|\left(K_{P}\right)_{x}\right\| \leqq \lambda<1$ and that $\left\|\left(I+\left(K_{P}\right)_{x}\right)^{-1}\right\| \leqq(1-\lambda)^{-1}$. By Lemma 4.2 and the remark following it we may write $K_{x}=B D_{x} C$ where for each $x\left\|D_{x}\right\| \leqq 1$. Hence $\left\|\left(K_{P}\right)_{x}\right\|_{1} \leqq\left\|K_{P x}\right\|_{1}=\left\|B D_{P x} C\right\|_{1} \leqq\|B\|_{2}\|C\|_{2}$. Thus by Lemma 4.1 it suffices to prove that $\left\|\left(K_{P}\right)_{x}-\left(K_{Q}\right)_{x}\right\|_{1}$ converges to zero in probability as $P$ and $Q$ approach $I$ in the directed set $\mathcal{F}$. Now

$$
\begin{aligned}
& \left\|\left(K_{P}\right)_{x}-\left(K_{Q}\right)_{x}\right\|_{1}=\left\|P K_{P x} P-Q K_{Q x} Q\right\|_{1} \\
& \quad \leqq\left\|(P-Q) K_{P x} P\right\|_{1}+\left\|Q K_{P x}(P-Q)\right\|_{1}+\left\|Q\left(K_{P x}-K_{Q x}\right) Q\right\|_{1} \\
& \quad \leqq\left\|(P-Q) B D_{P x} C\right\|_{1}+\left\|B D_{P x} C(P-Q)\right\|_{1}+\left\|K_{P x}-K_{Q x}\right\|_{1} \\
& \quad \leqq\|(P-Q) B\|_{2}\|C\|_{2}+\|B\|_{2}\|C(P-Q)\|_{2}+\left\|K_{P x}-K_{Q x}\right\|_{1} .
\end{aligned}
$$

The first two terms in the last line approach zero uniformly in $x$ as was proven in Lemma 1.2. The third term approaches zero in probability for observe that in the proof of Theorem 1 it was shown that $|f(P x)-f(Q x)|$ converges to zero in probability as $P$ and $Q$ approach $I$ through $\mathcal{F}$ where $f$ is the function satisfying the hypothesis of Theorem 1. By hypothesis (2) of the present theorem $\left\|K_{x}-K_{y}\right\|_{1}$ has exactly the same properties as $|f(x)-f(y)|$. Hence 
the first two paragraphs of the proof of Theorem 1 apply to the present case yielding the lemma.

Lemma 4.8. Let $T$ satisfy the hypothesis of the theorem. Let

$$
g_{P}(x)=\exp \left[\left(-2\left(K_{P} x, x\right)-\left\|K_{P} x\right\|^{2}\right) / 2 c\right] \operatorname{det}\left[\left(\left(T_{P}\right)_{x}{ }^{*}\left(T_{P}\right)_{x}\right)^{1 / 2}\right]
$$

for each finite dimensional projection $P$ in $\mathcal{F}$ where $K_{P}=P K P$ and $T_{P}=I+K_{P}$. Let $g$ be the function defined in the statement of the theorem. Then $g$ is uniformly continuous near zero in the topology $\mathrm{H}_{2}$ and if $\tilde{g}$ denotes the corresponding measurable function on $H$ with respect to the normal distribution then lim in probability ${ }_{P \rightarrow I} g_{P}=\tilde{g}$. Further, $\operatorname{Prob}(\tilde{g}>0)=1$.

Proof. Let $A=\left(B^{2}+C^{2}\right)^{1 / 2}$. By Lemma 4.4 and its proof $(K x, x)$ is uniformly continuous in $H_{2}$ on each of the sets $V_{n}=\{x:\|A x\|<n\}$ and is bounded on each such set. By Lemma 4.5 and its proof $\|K x\|^{2}$ is also uniformly continuous on each of the sets $V_{n}$. Since the exponential function is uniformly continuous on right bounded intervals the exponential factor in $g(x)$ is uniformly continuous in $H_{2}$ on each set $V_{n}$ and is also bounded on each $V_{n}$. The function $\operatorname{det}\left[\left(T_{x}^{*} T_{x}\right)^{1 / 2}\right] \equiv \Delta\left(K_{x}\right)$ is uniformly continuous near zero in $\mathrm{H}_{2}$ by Lemma 4.1, hypotheses (1) and (2) of the theorem and from the fact that $\left\|K_{x}\right\|_{1}=\left\|B D_{x} C\right\|_{1} \leqq\|B\|_{2}\|C\|_{2}$. If $B_{n}$ is a sequence of HilbertSchmidt operators such that $\left\|B_{n}\right\|_{2} \rightarrow 0$ and such that $\Delta\left(K_{x}\right)$ is uniformly continuous in $H_{2}$ on each of the sets $\left\{x:\left\|B_{n} x\right\|<1\right\}$ then putting $C_{n}^{2}=B_{n}^{2}+n^{-2} A^{2}$ it follows that $\left\|C_{n}\right\|_{2} \rightarrow 0$ as $n \rightarrow \infty$ and that $g(x)$ is uniformly continuous on each of the sets $\left\{x:\left\|C_{n} x\right\|<1\right\}$.

It follows from Lemmas 4.4 and 4.5 that the exponential factors in $g_{P}(x)$ and $g(P x)$ converge in probability to the same limit as $P \rightarrow I$ through $\mathcal{F}$. We shall show that $\Delta\left(\left(K_{P}\right)_{x}\right)$ and $\Delta\left(K_{P x}\right)$ also converge to the same limit. By virtue of Lemma 4.1 it suffices to show that the net $\left\|\left(K_{P}\right)_{x}-K_{P x}\right\|_{1}$ of tame functions converges to zero in probability. To this end observe that $\left\|\left(K_{P}\right)_{x}-K_{P x}\right\|_{1}=\left\|P K_{P x} P-K_{P x}\right\|_{1} \leqq\left\|(P-I) K_{P x} P\right\|_{1}+\left\|K_{P x}(P-I)\right\|_{1}$ $\leqq\left\|(P-I) B D_{P x} C P\right\|_{1}+\left\|B D_{P x} C(P-I)\right\|_{1} \leqq\|(P-I) B\|_{2}\|C\|_{2}+\|B\|_{2}\|C(P-I)\|_{2}$. Hence $\left\|\left(K_{P}\right)_{x}-K_{P x}\right\|_{1}$ converges to zero uniformly in $x$ as $P \rightarrow I$ through $\mathcal{F}$. All but the last assertion of the lemma is now proved. To prove that $\operatorname{Prob}(\tilde{g}>0)=1$ observe that $\tilde{g}$ is the product of two factors one of which is the exponential of an almost everywhere real valued function and the other is a limit of positive functions which by Lemma 4.8 are bounded away from zero.

Proof of theorem. Let $u\left(t_{1}, \cdots, t_{k}\right)$ be a continuous real valued function on $E_{k}$ with compact support. Define for each positive integer $j$

$$
h_{j}(t)=\left\{\begin{array}{cl}
1, & 0 \leqq t<j-1 \\
j-t, & j-1 \leqq t<j \\
0, & j \leqq t
\end{array}\right.
$$


Let $y_{1}, \cdots, y_{k}$ be in $H$ and define $f(x)=u\left(\left(x, y_{1}\right), \cdots,\left(x, y_{k}\right)\right)$. Then $f$ is a tame function on $H$. If $P$ is in the directed set $\mathcal{F}$ of finite dimensional projections on $H$ and $T_{P}=I+P K P$ then $f\left(T_{P} x\right)$ is a measurable tame function and by Lemma 4.3 and the remark following it and, by the continuity of $u, f\left(T_{P} x\right)$ converges in probability to $u\left(m\left(y_{1}\right), \cdots, m\left(y_{k}\right)\right)$ as $P$ approaches $I$ through $F h_{j}(\|A P x\|)$ is also a measurable tame function as is also $h_{j}\left(\left\|A P\left(T_{P}\right)^{-1} x\right\|\right)$. By Lemmas 1.2 and 4.6 and the continuity of $h_{j}$ it follows that $h_{j}(\|A P x\|)$ and $h_{j}\left(\left\|A P\left(T_{P}\right)^{-1} x\right\|\right)$ both converge in probability for each $j$ as $P \rightarrow I$ through $\mathcal{F}$ to measurable functions on $H$ with respect to the normal distribution which we denote respectively by $h_{j}^{\prime}(\|A(\cdot)\|)$ and $h_{j}^{\prime}\left(\left\|A T^{-1}(\cdot)\right\|\right)$.

Now if $L$ is a differentiable transformation of $E_{n}$ onto itself which is one to one and has a nonvanishing Jacobian, det $L_{x}$, then as is well known

$$
\begin{aligned}
\int_{E_{n}} v(x) \exp & \left(-\|x\|^{2} / 2 c\right) d x \\
& =\int_{E_{n}} v(L y) \exp \left[\left(\|y\|^{2}-\|L y\|^{2}\right) / 2 c\right]\left|\operatorname{det} L_{y}\right| \exp \left(-\|y\|^{2} / 2 c\right) d y
\end{aligned}
$$

if $v$ is continuous and has compact support. Noting that $\|x\|^{2}-\left\|T_{P} x\right\|^{2}$ $=-2(P K P x, x)-\|P K P x\|^{2}$ it follows that

$$
E\left(f\left(T_{P} x\right) h_{j}(\|A P x\|) g_{P}(x)\right)=E\left(f(x) h_{j}\left(\left\|A P\left(T_{P}\right)^{-1} x\right\|\right)\right)
$$

where $g_{P}$ is defined in Lemma 4.8. The integrand on the right side of (3) is bounded and converges in probability as $P \rightarrow I$ through $\mathcal{F}$, hence converges in mean to $u\left(n\left(y_{1}\right), \cdots, n\left(y_{k}\right)\right) h_{j}^{\prime}\left(\left\|A T^{-1}(\cdot)\right\|\right)$. Thus the right side of (3) converges to $E\left(f(\cdot) h_{j}^{\prime}\left(\left\|A T^{-1}(\cdot)\right\|\right)\right)$. By Lemma $4.4\left|\left(K_{P} x, x\right)\right| \leqq\|A P x\|^{2}$. It follows from this, from Lemma 4.7 and from the definition of $h_{j}$ that $h_{j}(\|A P x\|) g_{P}(x)$ is bounded' by $b \cdot \exp \left(j^{2} / c\right)$ for all $P$ in $\mathcal{F}$ and $x$ in $H$ where $b$ is given in Lemma 4.7. Thus the integrand on the left side of equation (3) is bounded and in view of Lemma 4.8 converges in mean as $P \rightarrow I$ through $F$. Passing to the limit in (3) we obtain

$$
\begin{aligned}
& E\left(u\left(m\left(y_{1}\right), \cdots, m\left(y_{k}\right)\right) h_{j}^{\prime}(\|A(\cdot)\|) \tilde{g}\right) \\
& \quad=E\left(u\left(n\left(y_{1}\right), \cdots, n\left(y_{k}\right)\right) h_{j}^{\prime}\left(\left\|A T^{-1}(\cdot)\right\|\right)\right) .
\end{aligned}
$$

Assume further that $u \geqq 0$. As $j \rightarrow \infty$ both $h_{j}^{\prime}(\|A(\cdot)\|)$ and $h_{j}^{\prime}\left(\left\|A T^{-1}(\cdot)\right\|\right)$ increase to the function identically equal to one. Application of the Lebesgue monotone convergence theorem to both sides of (4) yields

$$
E\left(u\left(m\left(y_{1}\right), \cdots, m\left(y_{k}\right)\right) \tilde{g}\right)=E\left(u\left(n\left(y_{1}\right), \cdots, n\left(y_{k}\right)\right) .\right.
$$

Let $u$ run through a sequence, $u_{j}$, of continuous functions on $E_{k}$ with compact supports such that $u_{j} \geqq 0$ and $u_{j} \uparrow 1$ monotonically. Again by the monotone convergence theorem we get $E(\tilde{g})=E(1)=1$. Hence $\tilde{g}$ is in $L^{1}(H)$ with respect to the normal distribution. Equation (5) may be extended to hold for 
non-negative bounded continuous functions, $u$, by the monotone convergence theorem and to all bounded continuous functions by consideration of their positive and negative parts. The set of all bounded real valued Baire functions on $E_{k}$ for which equation (5) holds is closed under bounded pointwise limits hence includes all bounded Baire functions.

By Lemma $4.8 \tilde{g}>0$ almost everywhere. Hence if $u\left(n\left(y_{1}\right), \cdots, n\left(y_{k}\right)\right)$ is a null function so is $\left|u\left(n\left(y_{1}\right), \cdots, n\left(y_{k}\right)\right)\right|$ and by (5) so is

$$
\left|u\left(m\left(y_{1}\right), \cdots, m\left(y_{k}\right)\right)\right| \text { and } u\left(m\left(y_{1}\right), \cdots, m\left(y_{k}\right)\right) \text {. }
$$

Conversely $u\left(m\left(y_{1}\right), \cdots, m\left(y_{k}\right)\right)=0$ a.e. implies $u\left(n\left(y_{1}\right), \cdots, n\left(y_{k}\right)\right)=0$ a.e. Furthermore $u\left(n\left(y_{1}\right), \cdots, n\left(y_{k}\right)\right)$ and $u\left(m\left(y_{1}\right), \cdots, m\left(y_{k}\right)\right)$ depend only on $f$ and $T$ and not on the particular choice of $y_{1}, \cdots, y_{k}$ and $u$. Hence the map $\phi_{0}: u\left(n\left(y_{1}\right), \cdots, n\left(y_{k}\right)\right) \rightarrow u\left(m\left(y_{1}\right), \cdots, m\left(y_{k}\right)\right)$ is a well defined isomorphism from the algebra $\mathfrak{N}_{0}$ of bounded complex valued measurable tame functions mod null functions on $H$ with respect to the normal distribution, $n$, onto the similarly defined algebra $\Re_{0}$ with respect to $m$. Let $\mathscr{N}$ and $\mathscr{T}$ be the bounded algebras of $H$ with respect to $n$ and $m$ respectively. To conclude the proof of the theorem it suffices to show that $\phi_{0}$ can be extended to an isomorphism from $\mathfrak{N}$ onto $\mathfrak{N}$ since such an isomorphism is always complete as was remarked in connection with Theorem $3 . \Re_{0}$ and $\mathfrak{M}_{0}$ are boundedly $L^{2}$ dense in $\mathscr{N}$ and $\mathfrak{T}$ respectively, i.e., every element of $\mathscr{N}$ (respectively $\mathfrak{N}$ ) is an $L^{2}$ limit of a uniformly bounded sequence of elements of $\mathfrak{T}_{0}$ (respectively $\mathfrak{T}_{0}$ ). If $f$ is in $\Re_{0}$ then (5) implies

$$
E\left(\phi_{0}(f) \tilde{g}\right)=E(f) .
$$

Hence if $f_{j}$ is a uniformly bounded sequence of functions in $\mathfrak{T}_{0}$ such that $E\left(\left|f_{j}\right|^{2}\right) \rightarrow 0$ as $j \rightarrow \infty$ then $E\left(\phi_{0}\left(\left|f_{j}\right|^{2}\right) \tilde{g}\right) \rightarrow 0$ as $j \rightarrow \infty$. Thus $\phi_{0}\left(\left|f_{j}\right|^{2}\right) \tilde{g} \rightarrow 0$ in probability and as $\tilde{g}>0$ a.e. $\phi_{0}\left(\left|f_{j}\right|^{2}\right) \rightarrow 0$ in probability hence in $L^{1}$. Thus $\phi_{0}$ is boundedly $L^{2}$ continuous and extends to a homomorphism, $\phi$, from $\mathfrak{N}$ into 9r. Note that (6) continues to hold for $\phi$ :

$$
E(\phi(f) \tilde{g})=E(f) \quad \text { for all } f \text { in } \Re .
$$

To show that $\phi(\Re)=\mathscr{N}$ it suffices to show that $\phi_{0}^{-1}$ is also boundedly $L^{2}$ continuous. This follows from (6) for if $\phi_{0}\left(f_{j}\right)$ is a uniformly bounded sequence of elements of $\mathfrak{M}_{0}$ with $f_{j}$ in $\Re_{0}$ such that $E\left(\left|\phi_{0}\left(f_{j}\right)\right|^{2}\right) \rightarrow 0$ as $j \rightarrow \infty$ then $\left|\phi_{0}\left(f_{j}\right)\right|^{2} \rightarrow 0$ in probability as does $\phi_{0}\left(\left|f_{j}\right|^{2}\right) \tilde{g}$ so that the bounded convergence theorem implies $E\left(\phi_{0}\left(\left|f_{j}\right|^{2}\right) \tilde{g}\right) \rightarrow 0$ and therefore $E\left(\left|f_{j}\right|^{2}\right) \rightarrow 0$. This concludes the proof of Theorem 4.

Corollary 4.1. Assume the hypothesis and notation of Theorem 4. The map $\phi_{0}: u\left(n\left(y_{1}\right), \cdots, n\left(y_{k}\right)\right) \rightarrow u\left(m\left(y_{1}\right), \cdots, m\left(y_{k}\right)\right)$ extends uniquely to an automorphism, $\phi$, of the bounded algebra, $\mathfrak{N}$, of $H$ with respect to the normal distribution. If $h$ is in $\mathfrak{N}$ then $E(\phi(h) \tilde{g})=E(h)$. Let a be the algebra of bounded complex valued functions on $H$ which are uniformly continuous near zero in the topology 
$H_{2}$. If $f$ is in a then $f \circ T$ is also in $a$ and the map $f \rightarrow f \circ T$ is an automorphism of a. Further,

$$
\phi(\tilde{f})=(f \circ T) \sim .
$$

There exists a unique unitary transformation $U$ of $L^{2}(H)$ such that $U(1)>0$ with probability 1 and $U M_{h} U^{-1}=M_{\phi(h)}$ where $M_{h}$ is the operator on $L^{2}(H)$ consisting of multiplication by the element $h$ of $\mathfrak{N}$.

The proof depends on the following lemma.

Lemma 4.1.1. Let $T$ be a transformation of $I$ into itself which is uniformly continuous near zero in the topology $\mathrm{H}_{2}$ with the $\mathrm{H}_{2}$ topology on its range. Assume further that $T$ has the following boundedness property: for any $\epsilon>0$ there exists $\delta>0$ with the property that if $D$ is a Hilbert-Schmidt operator with $\|D\|_{2}<\delta$ then there is a Hilbert-Schmidt operator $F$ with $\|F\|_{2}<\epsilon$ such that $\|D T x\|<1$ whenever $\|F x\|<1$. Let $f$ be a complex valued function on $H$ which is uniformly continuous near zero in the topology $H_{2}$. Then $f \circ T$ is also uniformly continuous near zero in $H_{2}$ and the net $\left\{(f \circ P \circ T)^{\sim}: P \in \mathcal{F}\right\}$ of measurable functions converges in probability to $(f \circ T)^{\sim}$ as $P \rightarrow I$ through $\mathcal{F}$.

Proof. Let $A_{n}$ be a sequence of Hilbert-Schmidt operators such that $\left\|A_{n}\right\|_{2} \rightarrow 0$ and such that $f$ is uniformly continuous in $H_{2}$ on each of the sets $V_{n}=\left\{x:\left\|A_{n} x\right\|<1\right\}$. Let $B_{n}$ be a sequence of Hilbert-Schmidt operators such that $\left\|B_{n}\right\|_{2} \rightarrow 0$ and such that $T$ is uniformly continuous from the topology $H_{2}$ to $H_{2}$ on each of the sets $W_{n}=\left\{x:\left\|B_{n} x\right\|<1\right\}$. For each positive integer $j$ let $\delta_{j}$ be a positive number with the property that if $\|D\|_{2}<\delta_{j}$ then there is a Hilbert-Schmidt operator $F$ with $\|F\|_{2}<1 / j$ such that $\|D T x\|<1$ whenever $\|F x\|<1$. Let $n_{j}$ be a sequence of positive integers such that $\left\|A_{n j}\right\|_{2}<\delta_{j}$ and let $F_{j}$ be a Hilbert-Schmidt operator such that $\left\|F_{j}\right\|_{2}<1 / j$ and such that $\left\|A_{n_{j}} T x\right\|<1$ whenever $\left\|F_{j} x\right\|<1$. Put $C_{j}^{2}=F_{j}^{*} F_{j}+B_{j}^{*} B_{j}$. Then $\left\|C_{j}\right\|_{2} \rightarrow 0$ and $T$ is uniformly continuous in $H_{2}$ on each set $U_{j}=\left\{x:\left\|C_{j} x\right\|<1\right\}$. Furthermore $f$ is uniformly continuous in $H_{2}$ on $T U_{j}$ since $\left\|C_{j} x\right\|<1$ implies $\left\|F_{j} x\right\|<1$ which implies $\left\|A_{n_{j}} T x\right\|<1$. Hence $f \circ T$ is uniformly continuous in $H_{2}$ on each set $U_{j}$ and is therefore uniformly continuous near zero in $H_{2}$.

Now if $P$ is a finite dimensional projection then $f \circ P$ is a continuous tame function. By the preceding paragraph $f \circ P \circ T$ is uniformly continuous near zero in the topology $H_{2}$. Thus we may form the measurable function $(f \circ P \circ T)^{\sim}$. Let $Q$ be in $\mathcal{F}$. If $\epsilon>0$ then

$$
\begin{aligned}
\operatorname{Prob}(|(f \circ P T) \sim-(f \circ T) \sim| \geqq \epsilon) \leqq & \operatorname{Prob}(|(f \circ P T) \sim-f \circ P T Q| \geqq \epsilon / 3) \\
& +\operatorname{Prob}(|f \circ P T Q-f \circ T Q| \geqq \epsilon / 3) \\
& +\operatorname{Prob}(|f \circ T Q-(f \circ T) \sim| \geqq \epsilon / 3) .
\end{aligned}
$$

The first and third terms on the right approach zero for each $P$ and $\epsilon$ as $Q \rightarrow I$ through $\mathcal{F}$. Hence it suffices to show that given $\delta>0$ there exists a 
projection $P_{0}$ in $\mathcal{F}$ such that if $P \geqq P_{0}$ then $\operatorname{Prob}(|f \circ P T Q-f \circ T Q| \geqq \epsilon / 3)<\delta$ for all $Q$ in $\mathcal{F}$. There exists a number $\alpha>0$ with the property that if $D$ is a Hilbert-Schmidt operator with $\|D\|_{2}<\alpha$ then there exists a Hilbert-Schmidt operator $F$ with $\|F\|_{2}^{2}<c^{-1} \delta / 3$ such that $\|D T x\|<1$ whenever $\|F x\|<1$. Choose $n$ such that $\left\|A_{n}\right\|_{2}<\alpha$. There exists a Hilbert-Schmidt operator $B$ such that $|f(x)-f(y)|<\epsilon / 3$ whenever $\left\|A_{n} x\right\|<1,\left\|A_{n} y\right\|<1$ and $\|B(x-y)\|$ $<1$. Thus $|f(P T Q x)-f(T Q x)|<\epsilon / 3$ whenever $\left\|A_{n} P T Q x\right\|<1,\left\|A_{n} T Q x\right\|<1$ and $\|B(I-P) T Q x\|<1$. Let $P_{0}$ be a finite dimensional projection such that $\|B(I-P)\|_{2}<\alpha$ whenever $P \geqq P_{0}$. Since $\left\|A_{n} P\right\|_{2}<\alpha$ it follows that if $P \geqq P_{0}$ there exist Hilbert-Schmidt operators $F_{P}, F$, and $G$ with Hilbert-Schmidt norms less than $\left(c^{-1} \delta / 3\right)^{1 / 2}$ such that $\left\|F_{P} Q x\right\|<1$ implies $\left\|A_{n} P T Q x\right\|<1$, $\|F Q x\|<1$ implies $\left\|A_{n} T Q x\right\|<1$ and $\|G Q x\|<1$ implies $\|B(I-P) T Q x\|<1$. Hence $|f(P T Q x)-f(T Q x)|<\epsilon / 3$ whenever $\left\|F_{P} Q x\right\|<1,\|F Q x\|<1$ and $\|G Q x\|<1$. Now $E\left(\|A Q x\|^{2}\right)=c\|A Q\|_{2}^{2}<\delta / 3$ when $A=F_{P}, F$, or $G$. Hence Prob $(\|A Q x\| \geqq 1)<\delta / 3$ when $A=F_{P}, F$ or $G$. Thus if $P \geqq P_{0}$ Prob $(|f(P T Q x)-f(T Q x)| \geqq \epsilon / 3)<\delta$ for all $Q$ in $\mathcal{F}$. This completes the proof of the lemma.

Proof of Corollary. In order to avoid possible confusion we shall distinguish in this proof between a continuous tame function, $f(x)$, on $H$ and the measurable tame function on the associated probability space to which $f$ corresponds. We shall denote the measurable tame function by $\tilde{f}$ as this is consistent with Theorem 1 . If $u\left(t_{1}, \cdots, t_{k}\right)$ is a continuous and bounded complex valued function on $E_{k}$ then

$$
\begin{aligned}
\phi_{0}\left(u\left(n\left(y_{1}\right), \cdots, n\left(y_{k}\right)\right)\right) & =u\left(\left(T(\cdot), y_{1}\right)^{\sim}, \cdots,\left(T(\cdot), y_{k}\right)^{\sim}\right) \\
& =u\left(\left(T(\cdot), y_{1}\right), \cdots,\left(T(\cdot), y_{k}\right)\right) \sim
\end{aligned}
$$

since convergence in probability of a net of random variables commutes with composition by a continuous function. Thus if $f$ is a continuous bounded tame function on $H$ then

$$
\phi_{0}(\tilde{f})=(f \circ T) \sim .
$$

We shall show that $T$ and $T^{-1}$ satisfy the hypothesis of Lemma 4.1.1. Put $S=T^{-1}$ and observe that $S$ has a Frechet derivative at each point $x$ in $H$ and that $S_{x}=\left(T_{S x}\right)^{-1}$. This follows from the inequality $\|T v-T u\|$ $\geqq(1-\lambda)\|v-u\|$ and from the relations

$$
\begin{aligned}
\| S y-S x & -\left(T_{S x}\right)^{-1}(y-x)\|/\| y-x \| \\
& =\left\|v-u-\left(T_{u}\right)^{-1}(T v-T u)\right\| /\|T v-T u\| \\
& \leqq\left\|\left(T_{u}\right)^{-1}\right\|\left\|T_{u}(v-u)-(T v-T u)\right\| /\|v-u\|(1-\lambda)
\end{aligned}
$$

wherein we have set $v=S y$ and $u=S x$. The last expression approaches zero as $v$ approaches $u$, hence as $y$ approaches $x$. Thus putting $u=S x$ we have 


$$
S_{x}=\left(I+K_{u}\right)^{-1}=I+\sum_{n=1}^{\infty}\left(-K_{u}\right)^{n}=I+B\left(-D_{u}+D_{u} C \sum_{n=0}^{\infty}\left(-K_{u}\right)^{n} B D_{u}\right) C
$$

where $B$ and $C$ are given in the statement of Theorem 4 and $\left\|D_{u}\right\| \leqq 1$ as in Lemma 4.2. Hence $\left|\left(\left(S_{x}-I\right) y, z\right)\right| \leqq\|C y\|\left\|B^{\prime} z\right\|$ where

$$
B^{\prime}=\left(1+(1-\lambda)^{-1}\|C\|\|B\|\right) B .
$$

Furthermore $S_{x}-I$ is a continuous function of $x$ with the uniform operator topology on the range. Hence $S_{x}-I$ has two properties similar to those of $K_{x}$. We shall prove that $T$ satisfies the hypothesis of Lemma 4.1.1 using only these two properties of $K_{x}$. The proof will therefore apply also to $T^{-1}=S$. Let $D$ be a Hilbert-Schmidt operator on $H$. In view of Lemma 4.2 and the remark following it we have $\|D T x\| \leqq\|D x\|+\|D K x\| \leqq\|D x\|+\left\|\int_{0}^{1} D B D_{t x} C x d t\right\|$ $\leqq\|D x\|+\|D B\|\|C x\|$. Put $F=2\left(D^{*} D+\|D B\|^{2} C^{2}\right)^{1 / 2}$. Then $F$ is a HilbertSchmidt operator and

$$
\begin{aligned}
\|F\|_{2}^{2} & \leqq 4\left(\|D\|_{2}^{2}+\|D B\|^{2}\|C\|_{2}^{2}\right) \\
& \leqq 4\|D\|_{2}^{2}\left(1+\|B\|^{2}\|C\|_{2}^{2}\right) .
\end{aligned}
$$

Furthermore $\|F x\|<1$ implies $\|D x\|<1 / 2$ and $\|D B\|\|C x\|<1 / 2$. Hence $\|D T x\|<1$ whenever $\|F x\|<1$. Given $\epsilon>0$ take $\delta=\epsilon / 2\left(1+\|B\|^{2}\|C\|_{2}^{2}\right)^{1 / 2}$. Thus if $\|D\|_{2}<\delta$ then $\|F\|_{2}<\epsilon$ and the boundedness assumption of Lemma 4.1.1 is proved. $T$ is uniformly continuous from $H_{2}$ to $H_{2}$ for if $D$ is a HilbertSchmidt operator then $\|D(T x-T y)\| \leqq\|D(x-y)\|+\|D(K x-K y)\|$ $\leqq\|D(x-y)\|+\|D B\|\|C(x-y)\|$ so that taking $F$ as before we have $\|D(T x-T y)\|<1$ whenever $\|F(x-y)\|<1$.

It was shown in the proof of Theorem 4 that $\phi_{0}$ extends to an isomorphism $\phi$ of $\mathscr{X}$ onto a subalgebra $\mathfrak{T}$ consisting of those functions in $\mathscr{N}$ which are measurable with respect to the smallest $\sigma$-ring with respect to which all the random variables $m(y), y \in H$ are measurable. By virtue of (7) $\phi$ is also boundedly $L^{2}$ continuous and therefore preserves convergence in probability of uniformly bounded nets of measurable functions in $\Re$. Hence if $f$ is an element of $a$ then $\phi(\tilde{f})=\lim$ in probability $P_{P \rightarrow I} \phi\left((f \circ P)^{\sim}\right)=\lim$ in probability $P \rightarrow I(f \circ P T)^{\sim}$ by $(8)$. Thus $\phi(\tilde{f})=(f \circ T)^{\sim}$ by Lemma 4.1.1.

Since $f \circ T^{-1}$ is in $a$ whenever $f$ is in $a$ by Lemma 4.1.1 it follows that the map $f \rightarrow f \circ T, f \in Q$, has $Q$ as its range and is therefore an automorphism of $Q$. Thus $\phi$ has in its range all the functions $\tilde{f}, f \in Q$, and in particular all continuous tame functions. Therefore $\mathfrak{N}=\mathfrak{N}$ and $\phi$ is an automorphism of $\Re$. Since an automorphism of $\mathscr{N}$ is necessarily boundedly $L^{2}$ continuous $\phi$ is unique. The existence and uniqueness of the unitary $U$ follows from the discussion preceding Theorem 3. This concludes the proof of the corollary.

THEOREM 5. Let $A$ be a transformation from a real Hilbert space $H$ into itself 
such that $A(0)=0$. Assume that $A$ has a Frechet derivative $A_{x}$ of trace class at each point $x$ in $H$ and that $A_{x}$ has the following properties:

(1) the map $x \rightarrow A_{x}$ is uniformly continuous near zero in the topology $\mathrm{H}_{2}$ with the trace class norm on the range.

(2) there exist Hilbert-Schmidt operators $B, C$ such that

$$
\left|\left(A_{x} y, z\right)\right| \leqq\|C y\|\|B z\|
$$

for all $x, y, z$ in $H$.

Then there exists a unique one parameter group of homeomorphisms $T_{t}$ of $H$ onto itself such that

(a) for each $x$ in $H, T_{t} x$ satisfies the differential equation

$$
d T_{t} x / d t=A T_{t} x, T_{0} x=x \quad \text { (strong derivative). }
$$

$T_{t}$ also has the following properties:

(b) There exists $a>0$ such that for $|t| \leqq a, T_{t}$ satisfies the hypothesis for $T$ in Theorem 4.

(c) $T_{t}: H_{2} \rightarrow H_{2}$ is uniformly continuous for each $t$.

(d) If $Q$ is the algebra of bounded complex valued functions on $H$ which are uniformly continuous near zero in the topology $H_{2}$ the maps $f \rightarrow f \circ T_{t}$ form a one-parameter group of automorphisms of $Q$. The maps $\tilde{f} \rightarrow\left(f \circ T_{t}\right)^{\sim}$ extend uniquely to a one-parameter group of automorphisms $\phi_{t}$ of the bounded algebra $\Re$ of $H$ with respect to the normal distribution. Finally, there exists a unique strongly continuous one-parameter group of unitary transformations $U_{t}$ on $L^{2}(H)$ such that $U_{t}(1)>0$ a.e. for each $t$ and

$$
M_{\phi_{t}(h)}=U_{t} M_{h} U_{t^{-1}}
$$

where $M_{h}$ is the operator on $L^{2}(H)$ consisting of multiplication by the element $h$ of $\mathfrak{N}$.

The proof depends on the following lemmas. We may assume without loss of generality that $B$ and $C$ are symmetric.

Lemma 5.1. If $A$ satisfies the hypothesis of the theorem then the equation $d T_{t} x / d t=A T_{t} x$ with initial condition $T_{0} x=x$ has a unique solution for each $x$. $T_{t} x$ is jointly continuous in $t$ and $x$ and the transformations $T_{t}$ form a oneparameter group of homeomorphisms of $H$.

Proof. By hypothesis (1) of the theorem the map $x \rightarrow A_{x}$ is continuous on $H$ with the operator norm on the range since $\left\|A_{x}\right\|_{1} \geqq\left\|A_{x}\right\|$. It follows from Lemma 4.2 and the remark following it that $A x-A y=\int_{0}^{1} A_{y+t(x-y)}(x-y) d t$. Hypothesis (2) of the theorem implies that $\left\|A_{x}\right\| \leqq\|B\|\|C\|$ so that $\|A x-A y\|$ $\leqq\|B\|\|C\|\|(x-y)\|$. Hence $A$ satisfies a uniform Lipschitz condition. The Cauchy-Lipschitz existence theorem may now be applied to yield the lemma.

We shall make use of the following lemma of Gronwall [6], the proof of which may be found in [8]. 
Gronwall's LemMa. Let $f(t)$ be a real valued function such that

$$
0 \leqq f(t) \leqq \lambda+\int_{0}^{t}(\mu f(s)+\nu) d s \quad \text { for } 0 \leqq t \leqq T
$$

where $\lambda, \mu, \nu$ are non-negative constants and $f$ is continuous on $[0, T]$. Then

$$
f(t) \leqq(\nu T+\lambda) \exp (\mu T) \quad 0 \leqq t \leqq T .
$$

Lemma 5.2. Let $K_{t}=T_{t}-I$. Then

$$
\left\|K_{t} x-K_{t} y\right\| \leqq\|B\|\|C(x-y)\||t| \exp (M|t|)
$$

where $M=\|B\|\|C\|$. Let $t>0$. For every Hilbert-Schmidt operator $E$ there exists a Hilbert-Schmidt operator $F$ such that $\left\|E\left(T_{8} x-T_{s} y\right)\right\| \leqq\|F(x-y)\|$ whenever $|s| \leqq t$ and such that $\|F\|_{2} \leqq d\|E\|_{2}$ where d depends only on $t$.

Proof. $T_{t} x-x=\int_{0}^{t} A T_{s} x d s$. Hence if $t \geqq 0$

$$
\begin{aligned}
\left\|K_{t} x-K_{t} y\right\| & \leqq \int_{0}^{t}\left\|A T_{s} x-A T_{s} y\right\| d s \\
& \leqq \int_{0}^{t}\left\|\int_{0}^{1} A_{T_{s} y+u\left(T_{s} x-T_{s} y\right)}\left(T_{s} x-T_{s} y\right) d u\right\| d s \\
\left.\leqq \int_{0}^{t} \int_{0}^{1}\left(\left\|A_{T_{s} y+u\left(T_{s} x-T_{s} y\right)}\left(K_{s} y-K_{s} x\right)\right\|+\| A_{T_{s} y+u\left(T_{s} x-T_{s} y\right.}\right)(x-y) \|\right) d u d s & \leqq \int_{0}^{t}\left(M\left\|K_{s} x-K_{s} y\right\|+\|B\|\|C(x-y)\|\right) d s .
\end{aligned}
$$

Here we have used Lemma 4.2 and the remark following it and condition (2) of the present theorem. Application of Gronwall's Lemma yields the first assertion of the lemma for $t \geqq 0$. For negative $t$ write $T_{-t} x-x=-\int_{0}^{t} A T_{-8} x d s$ with $t \geqq 0$ and proceed as before.

If $E$ is a Hilbert-Schmidt operator then

$$
\begin{aligned}
\left\|E\left(T_{s} x-T_{s} y\right)\right\| & \leqq\|E(x-y)\|+\left\|E\left(K_{s} x-K_{s} y\right)\right\| \\
& \leqq\|E(x-y)\|+\|E\|\|B\||s| \exp (M|s|)\|C(x-y)\| .
\end{aligned}
$$

Put $F^{2}=4\left(E^{*} E+\|E\|^{2}\|B\|^{2} t^{2} C^{2} \exp (2 M t)\right)$. Then $\left\|E\left(T_{\diamond} x-T_{\diamond} y\right)\right\| \leqq\|F(x-y)\|$ and

$$
\begin{aligned}
\|F\|_{2}^{2} & \leqq 4\|E\|_{2}^{2}+4\|E\|^{2}\|B\|^{2} t^{2}\|C\|_{2}^{2} \exp (2 M t) \\
& \leqq\|E\|_{2}^{2}\left(4+4\|B\|^{2} t^{2}\|C\|_{2}^{2} \exp (2 M t)\right)
\end{aligned}
$$

since $\|E\| \leqq\|E\|_{2}$. 
N.B. The proofs of the following lemmas will be carried out for $t \geqq 0$. The argument used in the preceding lemma may be applied to obtain the corresponding result for negative $t$.

Lemma 5.3. For each $t$ the Frechet derivative $\left(T_{t}\right)_{x}$ of $T_{t}$ exists and satisfies the equation

$$
d\left(T_{t}\right)_{x} / d t=A_{T_{t}}\left(T_{t}\right)_{x}, \quad\left(T_{0}\right)_{x}=I .
$$

The derivative is taken in the sense of the operator norm. Furthermore $\left\|\left(T_{t}\right)_{x}\right\|$ $\leqq \exp (M|t|)$.

Proof. For each $x$ let $S(t, x)$ be the operator valued solution of the equation

$$
d S(t, x) / d t=A_{T_{l} x} S(t, x), \quad S(0, x)=I .
$$

The solution exists and is unique by the Cauchy-Lipschitz theorem since $\left\|A_{T_{t} x}\right\| \leqq M=\|B\|\|C\|$ and $A_{T_{t} x}$ is a continuous function of $t$ in the operator norm for each $x$. $S$ satisfies the equation

$$
S(t, x)=I+\int_{0}^{t} A_{T_{s} x} S(s, x) d s .
$$

Upon taking the operator norm on both sides we obtain with the aid of Gronwall's Lemma $\|S(t, x)\| \leqq \exp (M|t|)$. Now

$$
\begin{aligned}
& T_{t} y-T_{t} x-S(t, x)(y-x)=(y-x)+\int_{0}^{t}\left(A T_{s} y-A T_{s} x\right) d s-S(t, x)(y-x) \\
& =\int_{0}^{t} \int_{0}^{1} A_{T_{s} x+u\left(T_{\bullet} y-T_{\triangleleft} x\right)}\left(T_{s} y-T_{s} x\right) d u d s-\int_{0}^{t} A_{T_{\mathrm{s}} x} S(s, x)(y-x) d s, \\
& =\int_{0}^{t}\left[\int_{0}^{1} A_{T_{s} x+u\left(T_{s} y-T_{s} x\right)}\left(T_{s} y-T_{s} x-S(s, x)(y-x)\right) d u\right. \\
& \left.+\int_{0}^{1}\left(A_{T_{\triangleleft} x+u\left(T_{\triangleleft} y-T_{\triangleleft} x\right)}-A_{T_{\triangleleft} x}\right) S(s, x)(y-x) d u\right] d s .
\end{aligned}
$$

Thus

$$
\begin{aligned}
\| T_{t} y- & T_{t} x-S(t, x)(y-x) \| \leqq \int_{0}^{t}\left(M\left\|T_{s} y-T_{s} x-S(s, x) y(y-x)\right\|\right. \\
& \left.+\|y-x\| e^{M T} \sup \left\{\left\|A_{T_{\triangleleft} x+u\left(T, y-T_{s} x\right)}-A_{T_{\imath} x}\right\|: 0 \leqq u \leqq 1,|s| \leqq T\right\}\right) d s
\end{aligned}
$$

for $t \leqq T$. Gronwall's Lemma now yields

$$
\begin{aligned}
& \left\|T_{t} y-T_{t} x-S(t, x)(y-x)\right\| \leqq\|y-x\| T \exp (2 M T) \\
& \cdot \sup \left\{\left\|A_{T_{\imath} x+u\left(T_{\imath} y-T_{\imath} x\right)}-A_{T_{x} x}\right\|: 0 \leqq u \leqq 1,|s| \leqq T\right\}
\end{aligned}
$$

for $0 \leqq t \leqq T$. By hypothesis (1) of the theorem there exist Hilbert-Schmidt 
operators $E$ with arbitrarily small Hilbert-Schmidt norm such that the map $z \rightarrow A_{z}$ is uniformly continuous in $H_{2}$ on the set $V=\{z:\|E z\|<1\}$. Choose such an $E$ with $\|E\|_{2}$ so small that the Hilbert-Schmidt operator $F$ whose existence is guaranteed by Lemma 5.2 with $t=T$ has the property that $\|F x\|<1 / 2$. Then $\left\|E T_{s} x\right\|<1 / 2$ for $|s| \leqq T$. Furthermore $\left\|T_{s} y-T_{s} x\right\|$ $\leqq$ const $\|y-x\|$ for $|s| \leqq T$ as follows from the first assertion of Lemma 5.2. Hence if $y$ is sufficiently close to $x$ in norm then $\left\|E\left(T_{s} x+u\left(T_{s} y-T_{s} x\right)\right)\right\|<1$ for $|s| \leqq T$ and $0 \leqq u \leqq 1$. It now follows from the uniform continuity of the map $z \rightarrow A_{z}$ on $V$ that the coefficient of $\|y-x\|$ on the right side of (9) approaches zero as $y \rightarrow x$. Thus $S(t, x)$ is the Frechet derivative of $T_{t}$ at $x$.

LeMma 5.4. For each $t,\left(K_{t}\right)_{x}=\left(T_{t}\right)_{x}-I$ is of trace class and the map $x \rightarrow\left(K_{t}\right)_{x}$ is uniformly continuous near zero in the topology $\mathrm{H}_{2}$ with the trace class norm topology on its range. Furthermore $\left\|\left(K_{t}\right)_{x}\right\|_{1} \leqq\|B\|_{2}\|C\|_{2}|t| \exp (M|t|)$.

Proof. By Lemma 5.3 the Frechet derivative of $T_{t}$ satisfies the equation

$$
\left(T_{\imath}\right)_{x}=I+\int_{0}^{t} A_{T_{8} x}\left(T_{s}\right)_{x} d s
$$

Hence $\left\|\left(K_{t}\right)_{x}\right\|_{1} \leqq \int_{0}^{t}\left\|A_{r_{x} x}\right\|_{1} \exp (M T) d s$ for $0 \leqq t \leqq T$. As $\left\|A_{T_{\mathbf{x}} x}\right\|_{1} \leqq\|B\|_{2}\|C\|_{2}$ it follows that $\left\|\left(K_{t}\right)_{x}\right\|_{1} \leqq\|B\|_{2}\|C\|_{2}|t| \exp (M|t|)$. Now

$$
\begin{aligned}
\left\|\left(K_{t}\right)_{x}-\left(K_{t}\right)_{y}\right\|_{1} & =\left\|\int_{0}^{t}\left(A_{T_{s} x}\left(T_{s}\right)_{x}-A_{T_{\imath} y}\left(T_{s}\right)_{y}\right) d s\right\|_{1} \\
& \leqq \int_{0}^{t}\left\|A_{T_{s} x}\left(\left(K_{s}\right)_{x}-\left(K_{s}\right)_{y}\right)\right\|_{1}+\left\|\left(A_{T_{s} x}-A_{T_{s} y}\right)\left(T_{s}\right)_{y}\right\|_{1} d s \\
& \leqq \int_{0}^{t} M\left\|\left(K_{s}\right)_{x}-\left(K_{s}\right)_{y}\right\|_{1}+\exp (M T)\left\|A_{T_{s} x}-A_{T_{\imath} \nu}\right\|_{1} d s
\end{aligned}
$$

for $0 \leqq t \leqq T$. Hence by Gronwall's Lemma (and replacing $T$ by $|t|$ at the end)

$$
\left\|\left(K_{t}\right)_{x}-\left(K_{t}\right)_{y}\right\|_{1} \leqq|t| \exp (2 M|t|) \sup \left\{\left\|A_{T_{b} x}-A_{T_{\diamond} \nu}\right\|_{1}:|s| \leqq|t|\right\} .
$$

Let $E_{n}$ be a sequence of Hilbert-Schmidt operators such that the map $z \rightarrow A_{z}$ is uniformly continuous in $H_{2}$ on each of the sets $V_{n}=\left\{z:\left\|E_{n} z\right\|<1\right\}$ with the trace class norm topology on its range. Let $F_{n}$ be the corresponding sequence of Hilbert-Schmidt operators whose existence is guaranteed by Lemma 5.2. Then $\left\|F_{n}\right\|_{2} \rightarrow 0$ and it follows from the above inequality and by using Lemma 5.2 once more that the map $x \rightarrow\left(K_{t}\right)_{x}$ is uniformly continuous in $H_{2}$ on each of the sets $W_{n}=\left\{x:\left\|F_{n} x\right\|<1\right\}$.

Lemma 5.5. $\left|\left(\left(K_{t}\right)_{x} y, z\right)\right| \leqq\|C y\|\|B z\||t| \exp (M|t|)$ for all $x, y, z$ in $H$ and $\left\|\left(K_{t}\right)_{x} y\right\| \leqq|t|\|B\|\|C y\| \exp (M|t|)$. 
Proof. It follows from the integral equation satisfied by $\left(T_{t}\right)_{x}$ that

$$
\begin{aligned}
\left\|\left(\left(T_{t}\right)_{x}-I\right) y\right\| & \leqq \int_{0}^{t}\left(\left\|A_{T_{s} x}\left(\left(T_{s}\right)_{x}-I\right) y\right\|+\left\|A_{T_{s} x} y\right\|\right) d s \\
& \leqq \int_{0}^{t}\left(M\left\|\left(\left(T_{s}\right)_{x}-I\right) y\right\|+\|B\|\|C y\|\right) d s .
\end{aligned}
$$

Hence Gronwall's Lemma now yields the second assertion of the lemma. Further,

$$
\begin{aligned}
\mid\left(\left(\left(T_{t}\right)_{x}\right.\right. & -I) y, z) \mid \leqq \int_{0}^{t}\left(\left|\left(A_{T_{s} x}\left(\left(T_{s}\right)_{x}-I\right) y, z\right)\right|+\left|\left(A_{T_{s} x} y, z\right)\right|\right) d s \\
& \leqq \int_{0}^{t}\left(\left\|C\left(\left(T_{s}\right)_{x}-I\right) y\right\|\|B z\|+\|C y\|\|B z\|\right) d s \\
& \leqq \int_{0}^{t}\left(\|C\|\|B\|\|C y\| s e^{M s}\|B z\|+\|C y\|\|B z\| e^{M s}\right) d s \text { since } e^{M s} \geqq 1, s \geqq 0 \\
& \leqq\|C y\|\|B z\| \int_{0}^{t}\left(M s e^{M s}+e^{M s}\right) d s \\
& \leqq\|C y\|\|B z\| t \exp (M t) .
\end{aligned}
$$

Lemma 5.6. Let $F$ be a Hilbert-Schmidt operator on $H$ such that $\|F\|<1$. Then the function $h(x)=\exp \left(\|F x\|^{2} / 2 c\right)$ is uniformly continuous near zero in the topology $\mathrm{H}_{2}$ and if $\tilde{h}$ denotes the corresponding measurable function on $\mathrm{H}$ with respect to the normal distribution (with parameter $c$ ) then $\tilde{h}$ is in $L^{1}(H)$.

Proof. $\|F x\|^{2}$ is uniformly continuous in $H_{2}$ on each set $V_{n}=\{x:\|F x\|<n\}$ and is bounded on each $V_{n}$. Hence $h(x)$ is uniformly continuous in $H_{2}$ on each $V_{n}$. The net $\|F P x\|^{2}$ of measurable tame functions converges in probability by Theorem 1 as $P \rightarrow I$ through $\mathcal{F}$ and as may be seen from Lemma 1.2 the same limit is attained by letting $P$ run through a suitable increasing sequence of spectral projections of $F^{*} F$. Thus we let $x_{1}, x_{2}, \cdots$ be an orthonormal basis of the ortho-complement of the null space of $F^{*} F$ such that $F^{*} F x_{n}=a_{n} x_{n}$ and let $P_{n}$ be the projection onto span $\left(x_{1}, x_{2}, \cdots, x_{n}\right)$. It follows that we may write $\tilde{h}=\lim$ in probability ${ }_{n \rightarrow \infty} h \circ P_{n}$. As the functions $h \circ P_{n}$ form an increasing sequence the monotone convergence theorem yields $E(h)$ $=\lim _{n \rightarrow \infty} E\left(h \circ P_{n}\right)$. Now

$$
\begin{aligned}
E\left(h \circ P_{n}\right) & =(2 \pi c)^{-n / 2} \int_{E^{n}} \exp \left(\sum_{i=1}^{n} a_{i} t_{i}^{2} / 2 c\right) \exp \left(\sum_{i=1}^{n} t_{i}^{2} / 2 c\right) d t_{1} d t_{2} \cdots d t_{n} \\
& =\prod_{i=1}^{n}\left(1-a_{i}\right)^{-1 / 2} .
\end{aligned}
$$

As $0<a_{i}<1$ and $\sum_{i=1}^{\infty} a_{i}<\infty$ the product converges as $n \rightarrow \infty$ concluding the proof of the lemma. 
Lemma 5.7. For all sufficiently small $t$ the function

$$
g_{t}(x)=\exp \left[\left(-2\left(K_{t} x, x\right)-\left\|K_{t} x\right\|^{2}\right) / 2 c\right] \operatorname{det}\left[\left(\left(T_{t}\right)_{x}{ }^{*}\left(T_{t}\right)_{x}\right)^{1 / 2}\right]
$$

is well defined and is uniformly continuous near zero in the topology $\mathrm{H}_{2}$. If $\tilde{g}_{t}$ denotes the corresponding measurable function on $H$ with respect to the normal distribution then $\tilde{g}_{t} \rightarrow 1$ in $L^{1}(H)$ as $t \rightarrow 0$.

Proof. By Lemmas 5.4 and 5.5, $K_{t}$ satisfies the hypothesis of Theorem 4 for sufficiently small $t$, say $|t|<a$. Hence for $|t|<a, g_{t}$ is well defined, i.e., $\Delta\left(\left(K_{t}\right)_{x}\right)$ is well defined and by Lemma $4.8 g_{t}$ is uniformly continuous near zero in $\mathrm{H}_{2}$.

By Lemma 5.4 $\left\|\left(K_{t}\right)_{x}\right\|_{1} \rightarrow 0$ uniformly in $x$ as $t \rightarrow 0$. Hence by Lemma 4.1 $\Delta\left(\left(K_{t}\right)_{x}\right) \rightarrow \Delta(0)=1$ uniformly in $x$ as $t \rightarrow 0$ and is bounded, say $\Delta\left(\left(K_{t}\right)_{x}\right) \leqq d$ for all $x$ and sufficiently small $t$. By Lemmas 5.5 and 4.2 and the remark following it we have

$$
\begin{aligned}
\left|-2\left(K_{t} x, x\right)-\left\|K_{t} x\right\|^{2}\right| & \leqq 2|t| e^{M|t|}\|C x\|\|B x\|+t^{2} e^{2 M|t|}\|C x\|^{2} \\
& \leqq b(t)\|F x\|^{2}
\end{aligned}
$$

where $F=\left(B^{2}+C^{2}\right)^{1 / 2}$ is Hilbert-Schmidt and $b(t) \rightarrow 0$ as $t \rightarrow 0$. Thus $g_{t}(x) \rightarrow 1$ uniformly on each of the sets $\|F x\|<n$ as $t \rightarrow 0$. Hence by Theorem $2 \tilde{g}_{t} \rightarrow 1$ in probability. Take $t_{0}$ so small that $b\left(t_{0}\right)\|F\|^{2}<1$ and put $h(x)$ $=d \exp \left(b\left(t_{0}\right)\|F x\|^{2} / 2 c\right)$. Then $\hbar$ is in $L^{1}(H)$ by Lemma 5.6 and $\tilde{g}_{t} \leqq \tilde{h}$ for $|t| \leqq t_{0}$ showing that $\tilde{g}_{t} \rightarrow 1$ in $L^{1}(H)$ by the dominated convergence theorem.

Proof of theorem. Assertion (a) of the theorem is contained in Lemma 5.1. Assertion (b) follows from Lemmas 5.4 and 5.5. Assertion (c) is contained in Lemma 5.2. For the proof of (d) observe that the map $f \rightarrow f \circ T_{t}$ is an automorphism of $a$ for sufficiently small $t$ by Corollary 4.1. Since for arbitrary $t, T_{t}$ is a product of such transformations with small $t$ the map $f \rightarrow f \circ T_{t}$ is an automorphism of $a$ for all $t$. A similar argument shows that for every $t$ the map $\tilde{f} \rightarrow\left(f \circ T_{t}\right)^{\sim}$ extends to a unique automorphism $\phi_{t}$ of $\Re$. Each automorphism $\phi_{t}$ corresponds to a unique unitary transformation $U_{t}$ on $L^{2}(H)$ as was shown in the discussion preceding Theorem 3 . It is easily verified that the $\phi_{t}$ and hence the $U_{t}$ form a one-parameter group. To conclude the proof of the theorem it remains to show that the one-parameter group of unitaries $U_{t}$ is strongly continuous. It suffices to prove the strong continuity at $t=0$.

Let $f$ be bounded and uniformly continuous in $H_{2}$. The set of measurable functions $\tilde{f}$ corresponding to such functions $f$ is dense in $L^{2}(H)$. It suffices to show that, for such $f, U_{t}(\tilde{f}) \rightarrow \bar{f}$ in $L^{2}(H)$ as $t \rightarrow 0$. Now

$$
\begin{aligned}
\left\|U_{t} \tilde{f}-\tilde{f}\right\| & =\left\|\left(f \circ T_{t}\right)^{\sim}\left(\tilde{g}_{t}\right)^{1 / 2}-\tilde{f}\right\| \\
& \leqq\left\|\left(f \circ T_{t}\right)^{\sim}\left(\left(\tilde{g}_{t}\right)^{1 / 2}-1\right)\right\|+\left\|\left(f \circ T_{t}\right)^{\sim}-\tilde{f}\right\| \\
& \leqq\|f\|_{\infty}\left\|\left(\tilde{g}_{t}\right)^{1 / 2}-1\right\|+\left\|\left(f \circ T_{t}\right)^{\sim}-\tilde{f}\right\|
\end{aligned}
$$


for sufficiently small $t$. We observe as in [16, Lemma 2.1] that the inequality $\left|a^{1 / 2}-b^{1 / 2}\right|^{2} \leqq|a-b|$ for non-negative real numbers $a, b$ implies that $\left\|\left(\tilde{g}_{t}\right)^{1 / 2}-1\right\|^{2} \leqq E\left(\left|\tilde{g}_{t}-1\right|\right)$ which converges to zero as $t \rightarrow 0$ by Lemma 5.7. It remains to show that $\left(f \circ T_{t}\right)^{\sim} \rightarrow \tilde{f}$ in $L^{2}(H)$. Since the functions $\left(f \circ T_{t}\right)^{\sim}$ are uniformly bounded it suffices to show that $\left(f \circ T_{t}\right)^{\sim} \rightarrow \tilde{f}$ in probability and for this, by Theorem 2 , it suffices to show that $f \circ T_{t} \rightarrow f$ uniformly on each of the sets $V_{n}=\{x:\|C x\|<n\}$. By Lemma 5.2, $\left\|T_{t} x-x\right\|$ $\leqq\|B\|\|C x\||t| \exp (M|t|)$. Hence $T_{t} x \rightarrow x$ uniformly on each $V_{n}$. As $f$ is uniformly continuous in $\mathrm{H}_{2}$ it is also uniformly continuous in the norm topology of $H$ so that $f\left(T_{t} x\right) \rightarrow f(x)$ uniformly on each $V_{n}$. This concludes the proof of the theorem.

Examples. (1) Let $B$ and $C$ be Hilbert-Schmidt operators on a real Hilbert space $H$. Suppose that $D$ is a Frechet differentiable transformation of $H$ into itself whose derivative $D_{x}$ is uniformly continuous on bounded subsets of $H$ in the norm topology of $H$ and with the operator norm on the range. If in addition $D(0)=0$ and $\left\|D_{x}\right\|$ is bounded then the transformation $A=B D C$ is easily verified to satisfy the hypothesis of Theorem 5 and if $\alpha$ is sufficiently small then $K=\alpha B D C$ satisfies the hypothesis of Theorem 4. An example of such a transformation $D$ is as follows. Put $R=(-\infty, \infty)$ and let $\phi(s, u)$ be defined on $R \times R$ and have the following properties:

(1) $\phi(s, 0)=0$;

(2) $\phi(s, u)$ is differentiable in $u$ for each $s$ and there exists a function $M(s)$ in real $L^{2}(R)$ such that

$$
\left|\phi_{u}(s, u)-\phi_{u}\left(s, u^{\prime}\right)\right| \leqq M(s)\left|u-u^{\prime}\right|
$$

for all $s, u, u^{\prime}$.

(3) For each $u, \phi_{u}(s, u)$ is Lebesgue measurable in $s$ and there exists a number $N$ such that for all measurable $f, \int_{-\infty}^{\infty}\left|\phi_{u}(s, f(s))\right| d s \leqq N$. Then the operator $D$ defined by

$$
(D f)(t)=\int_{-\infty}^{\infty} \phi(t-s, f(s)) d s
$$

is defined on $H=$ real $L^{2}(R)$, is Frechet differentiable and its Frechet derivative is a uniformly continuous function of $f$ with the operator norm on the range. We may prove this as follows. We note first that continuity of $\phi_{u}(s, u)$ in $u$ and measurability in $s$ imply measurability of $\phi_{u}(s, f(s))$ so that condition (3) makes sense. Writing

$$
\phi(t-s, f(s))=\int_{0}^{1} \phi_{u}(t-s, v f(s)) f(s) d v,
$$

we have 


$$
\begin{aligned}
|(D f)(t)|^{2} & =\left|\int_{-\infty}^{\infty} \int_{0}^{1} \phi_{u}(t-s, v f(s)) f(s) d v d s\right|^{2} \\
& \leqq\left(\int_{0}^{1} \int_{-\infty}^{\infty}\left|\phi_{u}(t-s, v f(s))\right|^{1 / 2}\left|\phi_{u}(t-s, v f(s))\right|^{1 / 2}|f(s)| d s d v\right)^{2} \\
& \leqq\left\{\int_{0}^{1}\left(\int_{-\infty}^{\infty}\left|\phi_{u}(t-s, v f(s))\right| d s\right)^{1 / 2}\right. \\
& \leqq N \int_{0}^{1} \int_{-\infty}^{\infty}\left|\phi_{u}(t-s, v f(s))\right||f(s)|^{2} d s d v .
\end{aligned}
$$

Hence

$$
\begin{aligned}
\int_{-\infty}^{\infty}|(D f)(t)|^{2} d t & \leqq N \int_{0}^{1} \int_{-\infty}^{\infty} \int_{-\infty}^{\infty}\left|\phi_{u}(t-s, v f(s))\right||f(s)|^{2} d t d s d v \\
& \leqq N^{2} \int_{-\infty}^{\infty}|f(s)|^{2} d s .
\end{aligned}
$$

Thus

$$
\|D f\|^{2} \leqq N^{2}\|f\|^{2} .
$$

It follows that $(D f)(t)$ is defined for almost all $t$ and that $D$ is defined on $H$. For each $f$ in real $L^{2}(R)$ the operator defined by

$$
\left(D_{f} g\right)(t)=\int_{-\infty}^{\infty} \phi_{u}(t-s, f(s)) g(s) d s
$$

is a bounded linear operator with $\left\|D_{f}\right\| \leqq N$ as may be seen from a calculation similar to the preceding one with the deletion of $\int_{0}^{1} d v$ wherever it appears and the replacement of $v$ by 1 . Further

$$
\begin{aligned}
\mid( & \left.D(f+g)-D(f)-D_{f} g\right)\left.(t)\right|^{2} \\
& =\left|\int_{-\infty}^{\infty}\left\{\int_{0}^{1} \phi_{u}(t-s, f(s)+v g(s)) g(s) d v-\phi_{u}(t-s, f(s)) g(s)\right\} d s\right|^{2} \\
& =\left|\int_{-\infty}^{\infty} \int_{0}^{1}\left\{\phi_{u}(t-s, f(s)+v g(s))-\phi_{u}(t-s, f(s))\right\} g(s) d v d s\right|^{2} \\
& \leqq \int_{0}^{1}\left[\int_{-\infty}^{\infty}\left|\phi_{u}(t-s, f(s)+v g(s))-\phi_{u}(t-s, f(s))\right|^{2} d s \int_{-\infty}^{\infty} g(s)^{2} d s\right] d v \\
& \leqq\|g\|^{2} \int_{0}^{1} \int_{-\infty}^{\infty}|M(t-s) v g(s)|^{2} d s d v .
\end{aligned}
$$


Hence

$$
\begin{aligned}
\left\|D(f+g)-D(f)-D_{f} g\right\|^{2} & \leqq\|g\|^{2} \int_{-\infty}^{\infty} \int_{-\infty}^{\infty} M(t-s)^{2} g(s)^{2} d t d s \\
& \leqq\|g\|^{2}\|M\|^{2}\|g\|^{2} .
\end{aligned}
$$

Thus $D_{f}$ is the Frechet derivative of $D$ at $f$. It remains to show that $D_{f}$ is uniformly continuous in $f$. This follows from the inequalities

$$
\begin{aligned}
\left|\left(D_{f}-D_{h}\right) g(t)\right|^{2} & =\left|\int_{-\infty}^{\infty}\left[\phi_{u}(t-s, f(s))-\phi_{u}(t-s, h(s))\right] g(s) d s\right|^{2} \\
& \leqq \int_{-\infty}^{\infty}\left|\phi_{u}(t-s, f(s))-\phi_{u}(t-s, h(s))\right|^{2} d s\|g\|^{2} \\
& \leqq\|g\|^{2} \int_{-\infty}^{\infty} M(t-s)^{2}|f(s)-h(s)|^{2} d s, \\
\left\|\left(D_{f}-D_{h}\right) g\right\|^{2} & \leqq\|g\|^{2} \int_{-\infty}^{\infty} \int_{-\infty}^{\infty} M(t-s)^{2}|f(s)-h(s)|^{2} d t d s \\
& \leqq\|g\|^{2}\|M\|_{2}^{2}\|f-h\|^{2} .
\end{aligned}
$$

Hence $\left\|D_{f}-D_{h}\right\| \leqq\|M\|_{2}\|f-h\|$.

As special cases of this example we may construct functions $\phi(s, u)$ having the above properties by setting $\phi(s, u)=\psi(s) \chi(u)$ where $\psi$ is in $L^{2}(R) \cap L^{1}(R)$ and $\chi$ is a differentiable function of $u$ which vanishes at zero and whose derivative is bounded and satisfies a uniform Lipschitz condition on $R$. In case $\chi(u)=u, D$ reduces to ordinary convolution.

(2) Another example of a transformation $D$ with the properties mentioned in the preceding example may be realized in the space $H=$ real $L^{2}(0,1)$. Let $\phi(t, s, u)$ be defined on $I \times I \times R$ where $I$ is the closed interval $[0,1]$. Assume that

(1) $\phi(t, s, 0)=0$.

(2) $\phi_{u}(t, s, u)$ exists for each $t, s, u$, is bounded in $t, s, u$ and satisfies

$$
\left|\phi_{u}(t, s, u)-\phi_{u}\left(t, s, u^{\prime}\right)\right| \leqq M\left|u-u^{\prime}\right|
$$

for all $t, s, u, u^{\prime}$ and for some constant $M$.

(3) For each $u, \phi_{u}(t, s, u)$ is a Borel measurable function on $I \times I$. Define $D$ by the relation

$$
(D f)(t)=\int_{0}^{1} \phi(t, s, f(s)) d s .
$$

Then $D$ is a Frechet differentiable transformation of $H$ into itself and its 
derivative $D_{f}$ is a uniformly continuous function of $f$ with the Hilbert-Schmidt norm on its range (and consequently with the operator norm on its range). To prove this observe that if $f$ is in $L^{2}(0,1)$ then $D f$ is also in $H$ by virtue of the inequalities

$$
\begin{aligned}
|(D f)(t)| & =\left|\int_{0}^{1} \int_{0}^{1} \phi_{u}(t, s, v f(s)) f(s) d v d s\right| \\
& \leqq N \int_{0}^{1}|f(s)| d s \\
& \leqq N\|f\|
\end{aligned}
$$

where $N=\sup \left|\phi_{u}(t, s, u)\right|$. Hence $\|D f\| \leqq N\|f\|$. A calculation similar to one in the preceding example shows that the Frechet derivative of $D$ at $f$ is given by $\left(D_{f} g\right)(t)=\int_{0}^{1} \phi_{u}(t, s, f(s)) g(s) d s . D_{f}$ is a Hilbert-Schmidt operator for each $f$ and has bounded Hilbert-Schmidt norm: $\left\|D_{f}\right\|_{2} \leqq N$. Finally we have

$$
\begin{aligned}
\left\|D_{f}-D_{h}\right\|_{2}^{2} & =\int_{0}^{1} \int_{0}^{1}\left|\phi_{u}(t, s, f(s))-\phi_{u}(t, s, h(s))\right|^{2} d t d s \\
& \leqq \int_{0}^{1} \int_{0}^{1} M^{2}|f(s)-h(s)|^{2} d t d s \\
& \leqq M^{2}\|f-h\|^{2} .
\end{aligned}
$$

(3) We may eliminate the transformation $B$ from the preceding example, i.e., we may take $A=D C$ and $K=\alpha D C$ if for the function $\phi(t, s, u)$ we write $\phi(t, s, u)=\psi(t, s) \chi(u)$ where $\psi(t, s)$ is bounded and measurable on $I \times I$ and $\chi(u)$ is a differentiable function which vanishes at 0 and whose derivative is bounded and satisfies a uniform Lipschitz condition. The uniform continuity of $A_{f}$ in $H_{2}$ with the trace class norm on the range follows from the uniform continuity of $D_{f}$ on $H$ with the Hilbert-Schmidt norm on the range proved above. Hypothesis (2) of Theorem 5 is satisfied by taking $B$ equal to $\left\|\chi_{u}\right\|_{\infty}$ times the adjoint of the operator with kernel $\psi(t, s)$.

(4) We shall indicate here the relation between our work and that of Cameron and Martin [2]. Let $H$ be real $L^{2}(0,1)$. We shall denote by $\mathfrak{e}$ the Wiener measure space consisting of the continuous functions on $[0,1]$ which vanish at zero. A representative of the normal distribution on $H$ with parameter $c=1$ is given by the stochastic integral; $n(f)(x)=\int_{0}^{1} f(t) d x(t)$. Here $f$ is in $H, x$ is in $\mathcal{C}$ and $n(f)$ is a random variable on $\mathcal{e}$ defined for almost all $x$ in $\mathfrak{e}$. Denote by $\mathfrak{e}^{\prime}$ the submanifold of $\mathfrak{e}$ consisting of those functions $x$ in $\mathfrak{e}$ which are absolutely continuous and whose derivative $x^{\prime}$ is square integrable on $[0,1]$. Let $D$ be the operator from $\mathfrak{e}^{\prime}$ onto $H$ defined by $D x=x^{\prime}$. Let $C$ be the operator from $H$ onto $\mathfrak{e}^{\prime}$ defined by $(C f)(t)=\int_{0}^{t} f(s) d s$. Then $C$ and $D$ are inverse to one another. We shall consider a transformation $S$ of $\mathfrak{e}$ onto $\mathcal{C}$ of the form $S: x \rightarrow x+\Lambda x$ where $\Lambda$ is a (not necessarily linear) transformation 
of $\mathfrak{C}$ into $\mathfrak{e}$ whose range is contained in $\mathfrak{C}^{\prime}$. These are the kind considered in [1] and [2]. Then $D \Lambda$ is a transformation of $\mathfrak{e}$ into $H$ and we shall assume that on each Hilbert sphere in $\mathcal{e}$ with center at the origin $D \Lambda$ is uniformly continuous from the Hilbert topology of $\mathfrak{e}$ to the weak topology of $H$. Now let $K=D \Lambda C$ and $T=D S C=I+K$. Then $T$ is a transformation of $H$ onto itself. $T$ and $S$ correspond to one another in the sense that when $T$ induces an automorphism of the bounded algebra of $H\left(=L^{\infty}(\mathfrak{e})\right)$ as in Theorem 4 then this automorphism is given by $F \rightarrow F \circ S$ for $F$ in $L^{\infty}(\mathcal{e})$. To prove this it suffices to show that for each $g$ in $H$ the net $\{(T P(\cdot), g) \sim$ of measurable tame functions (measurable functions on $\mathfrak{C}$ ) converges in probability to $n(g) \circ S$ as $P \rightarrow I$ through the directed set of finite dimensional projections on $H$. To this end it suffices to prove that the net $\{(K P(\cdot), g) \sim\}$ converges in probability to $n(g) \circ \Lambda$. Since $C$ is a Hilbert-Schmidt operator when the Hilbert space topology is taken on its range it follows that the function $(K(\cdot), g)$ is uniformly continuous near zero in the topology $H_{2}$. Hence the net $\left\{(K P(\cdot), g)^{\sim}\right\}$ of measurable functions on $\mathcal{e}$ converges in probability as $P \rightarrow I$. As has been remarked before the net of finite dimensional projections may be replaced by a sequence and in this case by a sequence of spectral projections of $C^{*} C$ where $C^{*}$ is the Hilbert space adjoint of $C$. A calculation (which we omit) shows that the functions $f_{k}(s)=2^{1 / 2} \cos \pi(k+1 / 2) s, k$ $=0,1,2, \cdots$ are the eigenfunctions of $C^{*} C$ and form an orthonormal basis of $L^{2}(0,1)$. Thus if we put $P_{k}$ equal to the projection of $H$ onto $\operatorname{span}\left\{f_{j}(s), j=0,1, \cdots, k\right\}$ we may write $\lim$ in $\operatorname{prob}_{P \rightarrow I}(K P(\cdot), g)^{\sim}$ $=\lim$ in $\operatorname{prob}_{k \rightarrow \infty}\left(K P_{k}(\cdot), g\right)^{\sim}$. If we write

$$
\left(K P_{k} f, g\right)=\phi\left(\left(f, f_{0}\right),\left(f, f_{1}\right), \cdots,\left(f, f_{k}\right)\right)
$$

then

$$
\left(K P_{k}(\cdot), g\right)^{\sim}=\phi\left(n\left(f_{0}\right), n\left(f_{1}\right), \cdots, n\left(f_{k}\right)\right) .
$$

Since the functions $f_{j}$ are differentiable the integrals $\int_{0}^{1} f_{j}(t) d x(t)$ exist for all $x$ in $\mathfrak{C}$ and we have

$$
\begin{aligned}
\left(K P_{k}(\cdot), g\right) \sim(x) & =\phi\left(n\left(f_{0}\right)(x), \cdots, n\left(f_{k}\right)(x)\right)=\left(K P_{k} \sum_{j=0}^{k} f_{j} n\left(f_{j}\right)(x), g\right) \\
& =\left(D \Lambda C \sum_{j=0}^{k} f_{j} n\left(f_{j}\right)(x), g\right) .
\end{aligned}
$$

Now

$$
\begin{aligned}
\left(C f_{j} n\left(f_{j}\right)(x)\right)(s) & =2 \int_{0}^{1} \cos \pi(j+1 / 2) t d t\left[\left.x(u) f_{j}(u)\right|_{0} ^{1}-\int_{0}^{1} x(u) f_{j}^{\prime}(u) d u\right] \\
& =2 \sin \pi(j+1 / 2) s \int_{0}^{1} x(t) \sin \pi(j+1 / 2) t d t .
\end{aligned}
$$


As the functions $2^{1 / 2} \sin \pi(j+1 / 2) s, j=0,1,2, \ldots$ form an orthonormal basis of $L^{2}(0,1)$ it follows that $C \sum_{j=0}^{k} f_{j} n\left(f_{j}\right)(x)$ converges in $L^{2}(0,1)$ to $x$ as $k \rightarrow \infty$. Hence $\left(K P_{k}(\cdot), g\right)^{\sim}(x)$ converges to $(D \Lambda x, g)$ as $k \rightarrow \infty$ since $D \Lambda$ is continuous from the Hilbert topology of $\mathfrak{e}$ to the weak topology of $H$. But as $(D \Lambda x, g)=n(g)(\Lambda x)$ the assertion that $(K P(\cdot), g)$ converges in probability to $n(g) \circ \Lambda$ is proved.

Rather than give further conditions on $\Lambda$ which will ensure that the transformation $T=I+D \Lambda C$ will satisfy the hypotheses of Theorem 4 and thereby induce an automorphism of $L^{\infty}(\mathcal{C})$ we shall merely give a class of examples of such transformations $\Lambda$ for which $T$ satisfies the hypotheses of Theorem 4 . To this end we consider the function $\phi(t, s, u)=\psi(t, s) \chi(u)$ considered in example (3) above. It is then readily verified that if $\alpha$ is sufficiently small we may take

$$
(\Lambda x)(t)=\alpha \int_{0}^{t} d v \int_{0}^{1} \phi(v, s, x(s)) d s
$$

for then the transformation $K=D \Lambda C$ is just the transformation of $H$ considered in example (3).

Comparison of this example with the results of [2] shows that we may obtain from Theorem 4 some transformations of $\mathfrak{e}$ not obtained in [2] which leave Wiener measure on $\mathcal{e}$ quasi-invariant, i.e., $w \circ S^{-1}$ is equivalent in the sense of absolute continuity to $w$ where $w$ is Wiener measure on $\mathrm{C}$. However we do not obtain all those transformations which are described in Theorem 2 of $[2]$.

\section{BIBLIOGRAPHY}

1. R. H. Cameron and W. T. Martin, Transformation of Wiener integrals under a general class of linear transformations, Trans. Amer. Math. Soc. vol. 58 (1945) pp. 184-219.

2. - The transformation of Wiener integrals by nonlinear transformations, Trans. Amer. Math. Soc. vol. 66 (1949) pp. 253-283.

3. K. O. Friedrichs, Mathematical aspects of the quantum theory of fields, New York, 1953.

4. K. O. Friedrichs and H. N. Shapiro, Integration over Hilbert space and outer extensions, Proc. Nat. Acad. Sci. U.S.A. vol. 43 (1957) pp. 336-338.

5. K. O. Friedrichs et al., Integration of functionals, New York University, Mimeographed notes. 1957.

6. T. H. Gronwall, Note on the derivatives with respect to a parameter of the solutions of a system of differential equations, Ann. of Math. vol. 20 (1919) pp. 292-296.

7. S. Kakutani, Determination of the spectrum of the flow of Brownian motion, Proc. Nat. Acad. Sci. U.S.A. vol. 36 (1950) pp. 319-323.

8. Solomon Lefschetz, Differential equations: geometric theory, New York, Interscience Publishers, Inc., 1957.

9. Karl Loewner, Grundzüge einer Inhaltslehre im Hilbertschen Raume, Ann. of Math. vol. 40 (1939) pp. 816-833.

10. E. R. Lorch, On the volume of smooth convex bodies in Hilbert space, Math. Z. vol. 61 (1955) pp. 391-407.

11. J. Milkman, Hermite polynomials, Hermite functionals and their integrals, in real Hilbert space, Riv. Mat. Univ. Parma vol. 6 (1955) pp. 65-68. 
12. R. Schatten, $A$ theory of cross spaces, Princeton University Press, 1950.

13. I. E. Segal, Decompositions of operator algebras II, Memoirs Amer. Math. Soc., no. 9, 1951.

14. - Tensor algebras over Hilbert spaces, Trans. Amer. Math. Soc. vol. 81 (1956) pp. 106-134.

15. - Abstract probability spaces and a theorem of Kolmogoroff, Amer. J. Math. vol. 57 (1954) pp. 721-732.

16. - Distributions in Hilbert space and canonical systems of operators, Trans. Amer. Math. Soc. vol. 88 (1958) pp. 12-41.

\author{
University of Chicago, \\ Chicago, Illinois \\ YALE UNIVERSITY, \\ New Haven, Connecticut
}

